EchoGéo

44 | 2018

Les valorisations territoriales et touristiques du street art

\title{
Valorisation territoriale du street art : le cas de la commune d'Abobo (Côte-d'Ivoire)
}

Ibrahima Sagnon, Hugues Ouattara Teninan and Félix Béchi Grah

\section{(2) OpenEdition}

\section{Journals}

Electronic version

URL: https://journals.openedition.org/echogeo/15717

DOI: 10.4000/echogeo.15717

ISSN: 1963-1197

Publisher

Pôle de recherche pour l'organisation et la diffusion de l'information géographique (CNRS UMR 8586)

Electronic reference

Ibrahima Sagnon, Hugues Ouattara Teninan and Félix Béchi Grah, "Valorisation territoriale du street art: le cas de la commune d'Abobo (Côte-d'Ivoire)", EchoGéo [Online], 44 | 2018, Online since 31 July 2018, connection on 31 July 2021. URL: http://journals.openedition.org/echogeo/15717 ; DOI: https:// doi.org/10.4000/echogeo.15717

This text was automatically generated on 31 July 2021

EchoGéo est mis à disposition selon les termes de la licence Creative Commons Attribution - Pas d'Utilisation Commerciale - Pas de Modification 4.0 International (CC BY-NC-ND) 


\title{
Valorisation territoriale du street art: le cas de la commune d'Abobo (Côte-d'Ivoire)
}

\author{
Ibrahima Sagnon, Hugues Ouattara Teninan and Félix Béchi Grah
}

\section{Introduction}

1 Fedel désigne le street art comme étant un phénomène mondial né dans les années 1960 aux États-Unis, recouvrant les pratiques artistiques les plus diverses : calligraphie au marqueur ou à la bombe aérosol, affiche, pochoir, peinture murale (Fedel, 2013). Pour Gorri, l'art urbain, récemment renommé street art, est un mouvement artistique contemporain qui regroupe toutes les formes d'art réalisées dans la rue ou dans des endroits publics et englobe diverses méthodes telles que le graffiti, le pochoir, la mosaïque, les stickers ou les installations. C'est principalement un art éphémère, un art public, illégal et officieux (Gorri, 2011). Selon Yang, les motivations qui poussent les artistes à afficher leur art dans la rue sont des plus variées : parfois pour signifier un mécontentement face à un fait de société, parfois pour passer un message percutant. Cette forme d'expression repose sur l'idée que l'artiste intervient dans l'espace public et sur des supports publics ou privés, qui ne lui appartiennent pas (Yang, 2014). Originellement, il s'agit de l'expression d'une revendication de liberté et d'une révolte contre le système politique. Au lendemain des années 2000 , face à une répression et un rejet de plus en plus important, le monopole de la signature laisse place à une plus grande diversité des interventions (Fedel, 2013). De nouvelles pratiques apparaissent avec la peinture aérosol de Banksy, Revs, JR ; avec la photographie et l'affichage, Space Invader ; avec la mosaïque, Atlas ; avec le Scotsh tricot (collectif tricot). Ainsi, le street art est un art en perpétuel renouvellement alimenté par les évolutions sociétales. Cet art qui durant de nombreuses années fût considéré comme illégitime et indigne du marché de l'art, davantage assimilé à de la délinquance qu'à la création, contestataire et parfois violent, se voit aujourd'hui considéré comme un art à part entière que les mairies et autres musées tentent de réguler en proposant des espaces d'expression bien 
définis. Le street art acquiert donc progressivement ses lettres de noblesse, ceci au-delà même des frontières des États-Unis, notamment en Côte d'Ivoire. Située au nord de la capitale économique ivoirienne (Abidjan), la commune d'Abobo se distingue par une diversité d'expressions artistiques de rue, notamment des fresques et des installations lumineuses. Accompagnant la chronologie du contexte politique ivoirien, on passe d'une pratique relevant de la jeunesse des quartiers populaires d'Abobo à une pratique encadrée par des institutions. Illégal et réprimandé à l'origine, le street art s'institutionnalise et se complimente. Cet article se propose donc de s'intéresser aux logiques qui sous-tendent cette institutionnalisation du street art à Abobo.

Pour ce faire, il convient de porter attention aux formes d'art réalisées, à leurs caractéristiques ainsi qu'aux stratégies de leur localisation et aux ressorts de leur production, afin de les mettre en relation avec l'évolution du contexte politique ivoirien. L'approche par l'image est celle adoptée pour l'étude. Les photographies qui constituent l'épine dorsale de cet article sont prises dans trois quartiers abritant les formes d'art urbain. Parallèlement, vingt-sept entretiens ont eu lieu avec les initiateurs de l'art urbain ainsi qu'avec des membres de la jeunesse d'Abobo - ceci tout en précisant que certaines informations ont dû être recueillies par personnes interposées, tant il est difficile de retrouver ou de contacter ces initiateurs. Les propos recueillis contribuent à mettre en lumière les logiques qui sous-tendent le processus d'institutionnalisation du street art. La carte ci-après présente les quartiers où l'enquête a été conduite pour l'observation de ces formes d'art.

Illustration 1 - Quartiers de street art

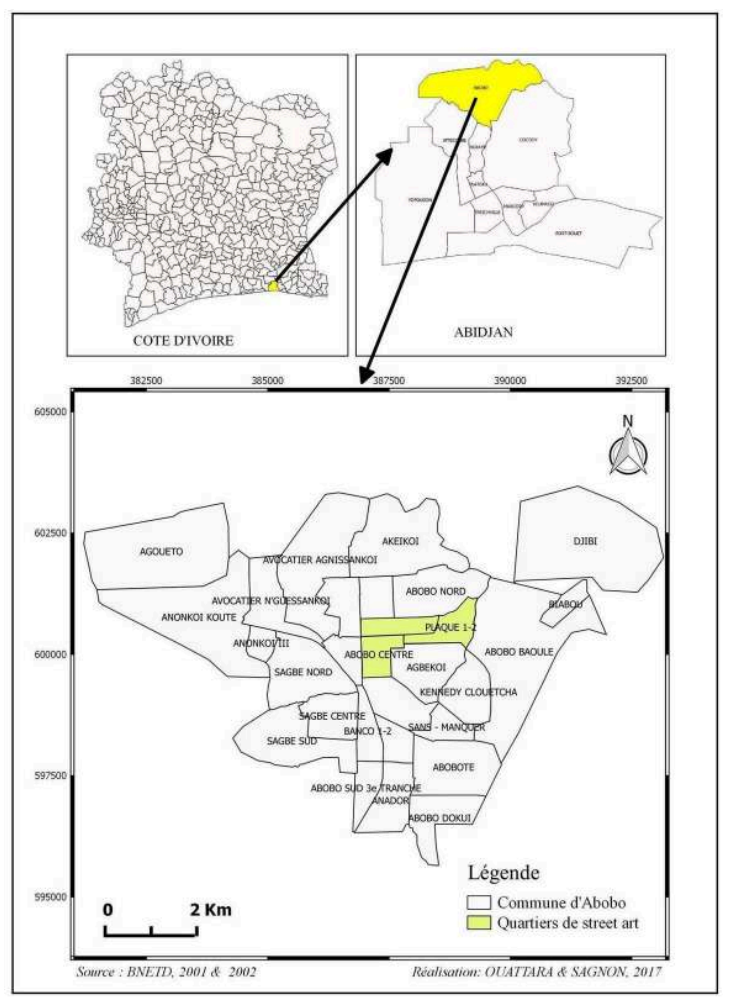

Les quartiers où l'enquête a été conduite à des fins d'observation des pratiques liées au street art de 2000 à 2014.

Auteur : I. Sagnon. 
Premier président de la république de Côte d'Ivoire, Félix Houphouët Boigny a gouverné le pays dès son accession à l'indépendance en 1960. Il fut le fondateur du Parti Démocratique de Côte d'Ivoire (PDCI). Après le décès de Houphouët Boigny en 1993, Henry Konan Bédié, membre héritier du PDCI, a assuré l'intérim du pouvoir présidentiel ivoirien. Ce dernier fut renversé du pouvoir par le général Robert Gueï lors d'un coup d'État orchestré en décembre 1999. Suite à ce putsch militaire, la Côte d'Ivoire a connu une perturbation de ses ministères, particulièrement celui de l'éducation nationale. À partir du premier mois de l'année 2000, le système éducatif est confronté à l'absence d'enseignants du fait de cette instabilité sociopolitique, engendrant par ricochet l'arrêt des cours et la perturbation des programmes. Pendant ces moments de troubles, des élèves de 15-20 ans ont exprimé leur mécontentement sur des bâtiments publics. Ils y trouvèrent le lieu idéal pour revendiquer leur droit à l'éducation comme en témoignent les propos ci-après de trois élèves du lycée moderne d'Abobo en l'an 2000 :

«Pour que les revendications soient prises en compte, il faille s'approprier les rues et espaces publics. »

" Mais, sachant qu'il s'agit du général Rober Guéi à la tête du pouvoir, il est préférable d'agir dans la clandestinité au risque de se faire arrêter par les forces de l'ordre. »

«Personne ne viendra balayer chez nous à notre place. À nous de prendre notre destin en main et de revendiquer nos droits particulièrement éducatifs, il y va de notre avenir ».

Ces élèves, non sans esprit de révolte à l'image de leur quartier à l'époque, adoptent une technique qu'ils dénomment le gbôchôli. Ce terme signifie " écriture » en nouchi, le langage né dans les rues et les espaces publics (marchés, gares routières) de la métropole abidjanaise précisément dans les quartiers à forte présence de déscolarisés et d'analphabètes comme à Abobo. Cette forme d'expression, composée de termes inventés par eux-mêmes, leur permet de contourner les fautes de grammaire et de conjugaison. Ces termes, parfois combinés avec des mots de la langue française, leurs permettent également de s'identifier géographiquement, au niveau socioprofessionnel et surtout de communiquer sans risque d'être compris par les gens de l'extérieur. Les élèves, au contact des fondateurs du nouchi à Abobo, leur ont emprunté ce langage qui petit à petit en est venu à être parlé par d'autres couches sociales. Les élites ivoiriennes vont ainsi adhérer à ce langage de rue. Et, par l'écriture, étape préliminaire d'apprentissage scolaire, les élèves expriment leur volonté de retourner à l'école et leur droit à l'éducation. Le gbôchôli se caractérise par des signatures constituées de noms souvent accompagnés de chiffres. Celles que présentent les illustrations 2 à 5 constituent des vestiges du gbôchôli dans la mesure où la quasi-totalité des murs qui ont fait l'objet de cette technique d'expression est aujourd'hui peint en une couleur unique. Elles présentent le gbôchôli en tant que technique d'art urbain, au quartier Plaque, dans la commune d'Abobo. 
Illustration 2 - Différentes formes de gbôchôli en 2000

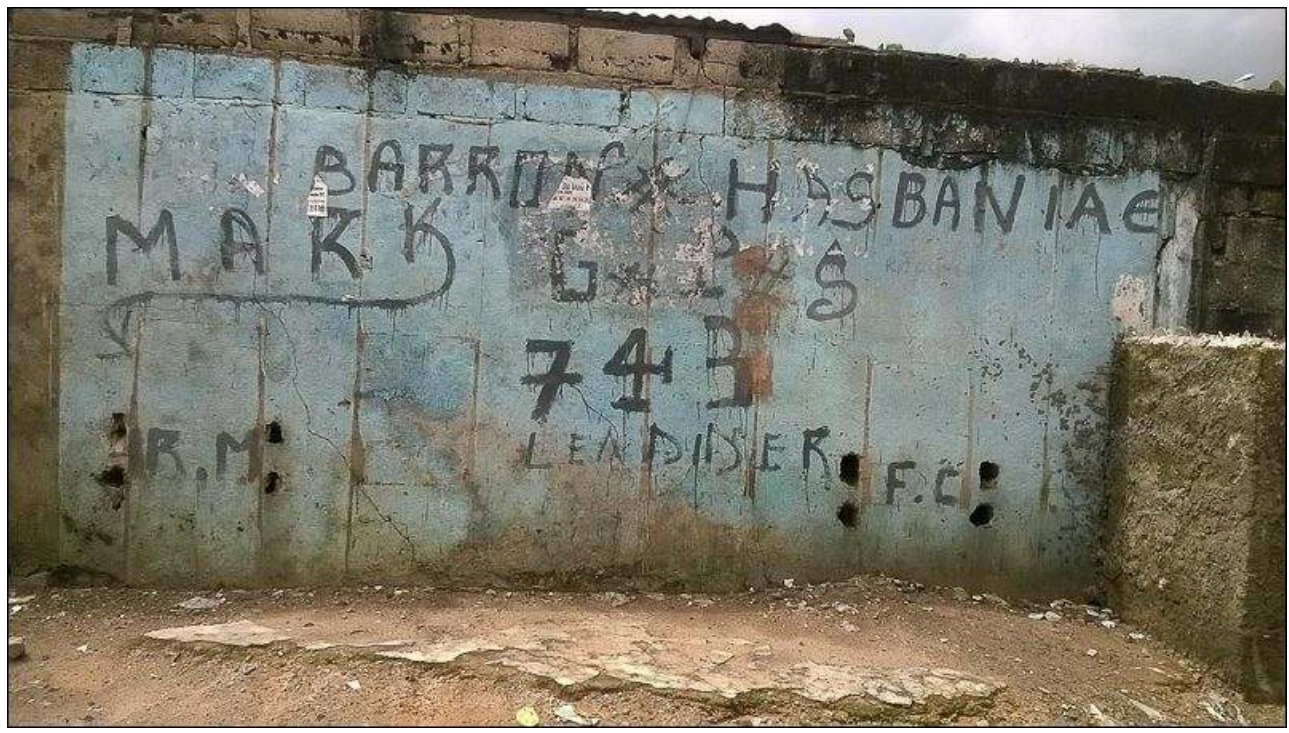

Le gbôchôli se présente sous différentes formes. II se constitue parfois avec des lettres, parfois des chiffres, parfois même les deux combinés.

Auteur : I. Sagnon, 2017.

Illustration 3 - Le gbôchôli sur le mur des maisons en 2000

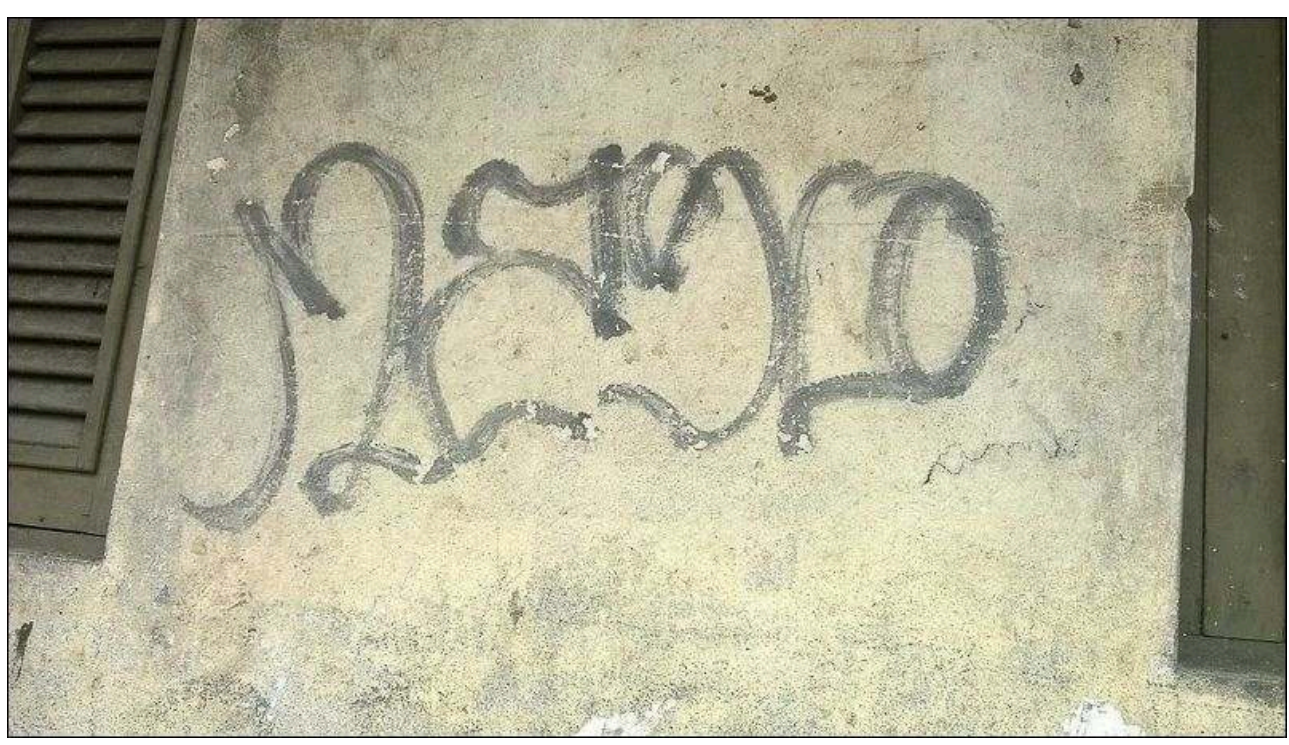

Le style arrondi de l'écriture est une marque d'appartenance à un cercle d'amis. Comme le présente cette image, le mur de logement au quartier Plaque constitue le support préférentiel pour le gbôchôli. Auteur: I. Sagnon, 2017. 
Illustration 4 - Vestige du gbôchôli

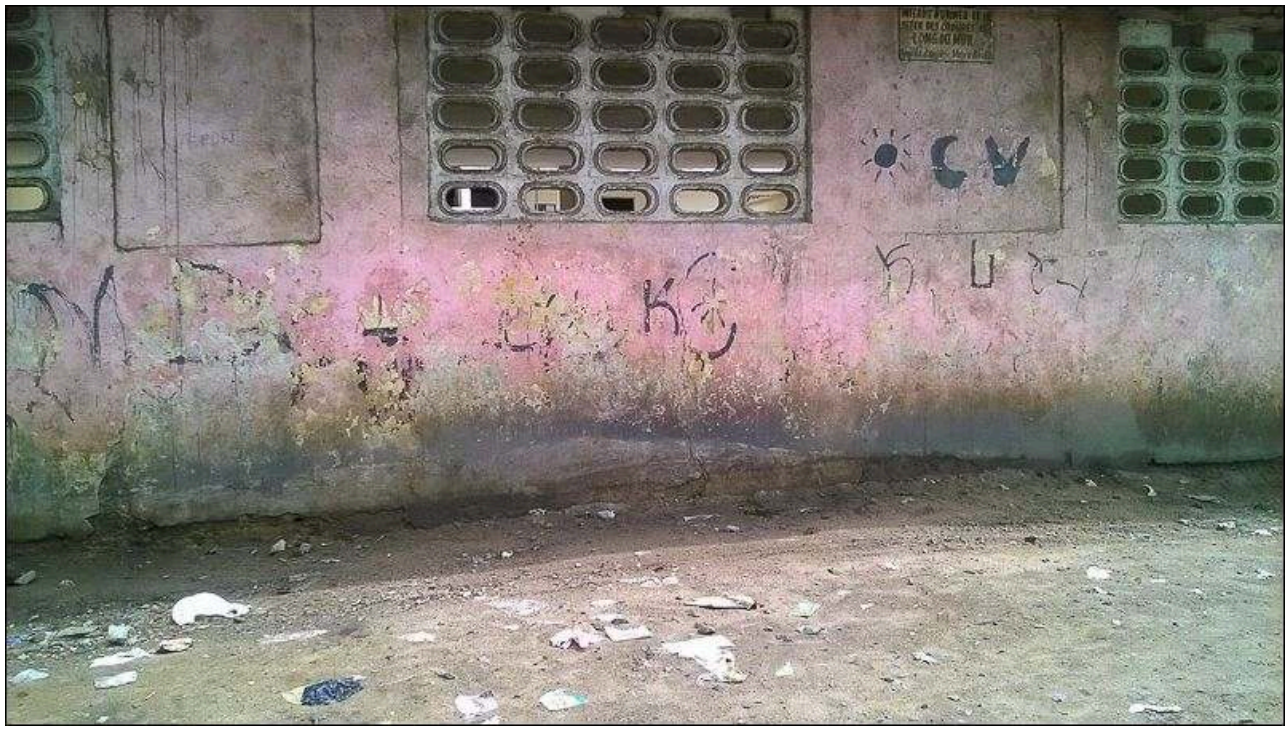

Interdite et réprimandée par la police ivoirienne en 2000, cette forme d'art urbain disparaît de l'habitude des jeunes élèves initiateurs. Cette image prise en 2017 représente ainsi un vestige difficilement visible du gbôchôli inscrit sur le mur de l'école Saint Etienne.

Auteur : I. Sagnon, 2017.

\section{Illustration 5 - Le gbôchôli, une expression de revendication}

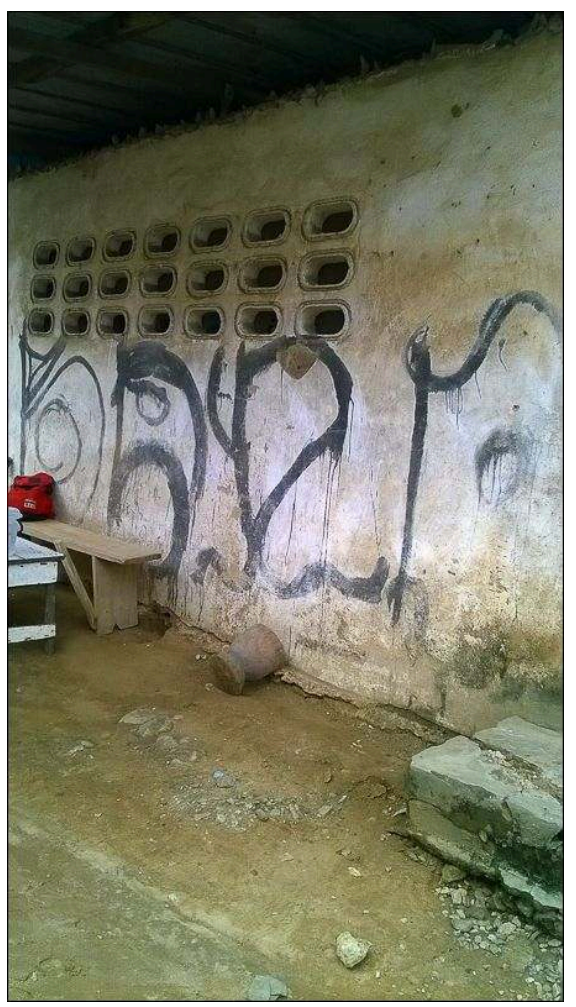

Inscrit sur le mur de l'école Saint Etienne au quartier Plaque, les jeunes élèves revendiquent leur droit à l'éducation. Suite à l'arrêt des cours induit par un coup d'État, ces élèves expriment leur ras-le-bol et leur volonté de retrouver les classes.

Auteur: I. Sagnon, 2017. 
5 Au-delà de l'expression de contestation et de revendication, le gbôchôli représente pour les élèves un outil de valorisation et de reconnaissance. Il permet à ces derniers de mettre à l'épreuve leur capacité esthétique par l'écriture et de souligner leur appartenance à un cercle d'amis. À l'aide d'un chiffon de matelas et de peinture à l'huile de couleur noire, les élèves investissent les murs des maisons (illustration 2 et 3 ) et ceux de l'école Sainte Étienne (illustration 4 et 5). Ils opèrent dans la clandestinité, de préférence de nuit, afin d'échapper à la vigilance de la police, des parents et des propriétaires des bâtiments. Tandis que certains font usage de chiffres, d'autres utilisent des lettres. Au travers de l'illustration 2, il est possible de lire "BARRON », faisant référence au baron qui signifie "personne importante", renvoyant à l'importance de l'auteur dans le cercle d'amis auquel il appartient. On aperçoit aussi le nom "MARK » avec la dernière lettre se transformant en une flèche, se référant au caractère d'une personne toujours prête à attaquer. Il est possible également de lire sur l'illustration 3 le nom « MASCO ». Dans son écriture, l'auteur emploie un style arrondi. C'est également une marque de reconnaissance entre membres d'un groupe d'amis. Toutes les signatures du gbôchôli ne sont pas aisées à décrypter. Tel est le cas des signatures figurant sur les illustrations 4 et 5. Questionné en l'an 2000 sur l'objectif et le support d'expression du gbôchôli, l'éducateur (46 ans) du collège se prononce où sont portées ces inscriptions précise :

«Bien vrai qu'il faut revendiquer ses droits, mais s'approprier des bâtiments de façon illégale n'est pas juste. D'autant plus que la technique d'expression (gbôchôli) encombre les murs, défigure le paysage urbain en laissant paraître un décor tacheté et incompréhensible ».

6 Cette interprétation du gbôchôli est partagée par les trois quarts de la population rencontrée. N'ayant ainsi pas rencontré l'adhésion de la population ivoirienne, le gbôchôli a perdu son élan et a fini par disparaitre des pratiques des élèves.

7 Dans le contexte de crise politique et militaire qui régnait au début des années 2000 , la Côte d'Ivoire voit se multiplier l'emprise des espaces publics où se tiennent des débats politiques, notamment dans la commune administrative du Plateau sur le modèle de la "Sorbonne ${ }^{1}$ ", comme aussi dans les communes populaires de Yopougon et Abobo. Ces espaces publics ou encore "parlements de rue ", sont fréquentés par des étudiants syndicalistes de la $\mathrm{FESCI}^{2}$ à partir de la campagne électorale de 2000, qui se solde par la victoire de Laurent Gbagbo (président du parti politique dénommé Front Populaire Ivoirien) sur le putschiste Robert Gueï. Alliés patriotiques du président Laurent Gbagbo, ces étudiants diffusent au sein de ces "parlements de rue», de virulentes paroles nationalistes. Ils s'imposent de façon hégémonique dans le Sud de la Côte d'Ivoire par l'entremise d'orateurs qui ont fait de cette prise de parole patriotique un véritable métier (Banégas., 2012). Dans cette situation, une rébellion est initiée en 2002 par des groupes armés venus du Nord du pays. Ils s'engagent à chasser le président du pouvoir comme le rapporte ci-après un partisan de la révolte (32 ans), rencontré en 2002 dans la rue : «Trop c'est trop ! On est fatigué. Laurent Gbagbo doit quitter le pouvoir ».

8 Les ressortissants du Nord du pays s'allient ensuite au parti de l'opposition, à savoir le Rassemblement Des Républicains (RDR) dont le président est Alassane Ouattara, lui aussi ressortissant de cette partie de la Côte d'Ivoire. Après plusieurs années de combats et d'accords de sortie de crise, une élection présidentielle est prévue en octobre 2010. Durant la campagne électorale, les partisans du RDR d'Alassane Ouattara et du FPI de Laurent Gbagbo (ou de la FESCI) expriment leur engagement de 
conquérants face à ce qu'ils considèrent être une usurpation du pouvoir présidentiel. Les propos "On gagne ou on gagne ", recueillis en 2010 auprès de jeunes partisans du RDR, signifient qu'ils sont prêts à tout sacrifier même leur vie afin de permettre au RDR et à Alassane Ouattara d'accéder à la magistrature suprême. Des partisans du FPI, aussi jeunes que leurs rivaux politiques du RDR, s'expriment à travers les termes «Ya rien en face, c'est maïs ». Rencontrés dans la rue pendant la campagne électorale, ils affirment leur sentiment de supériorité. Pour ces derniers, personne ne peut empêcher leur parti politique (FPI) de remporter l'élection présidentielle.

Ces propos révèlent l'intérêt capital que les partisans de ces deux formations politiques accordent au pouvoir présidentiel. Mais un intérêt exprimé verbalement qui pourrait dégénérer et conduire à des affrontements. Cette crainte suscite l'attention et l'implication de l'Institut National Supérieur des Arts et de l'Action Culturel (INSAAC) dans la quête de la cohésion et de la paix nationale.

\section{L'institutionnalisation du graffiti à la quête de la cohésion nationale en 2010}

Dans l'optique d'apaiser l'atmosphère surchauffé pendant la campagne électorale de 2010 et d'éviter une éventuelle crise post-électorale, 12 étudiants dont l'âge est compris entre 19 et 27 ans, de la $3^{\mathrm{e}}$ et la $5^{\mathrm{e}}$ année de peinture de l'INSAAC, furent sollicités par l'agence des États-Unis pour le Développement International (USAID). Ayant pour mission de promouvoir la démocratie partout dans le monde, l'USAID a choisi des étudiants appartenant au FPI, au RDR et au PDCI sans discrimination. Ces étudiants, résidant à Abobo, Cocody et Yopougon, ont investi le mur de la cité estudiantine durant 10 jours. Ils ont procédé à un graffiti en scénettes à l'aide de pinceaux, rouleaux, seaux, serviettes, gants et de 150 pots de peintures d'un poids de $30 \mathrm{~kg}$ chacun, rouge, bleu, blanc, vert et jaune. À travers les images peintes, ces étudiants avaient pour objectif de sensibiliser les populations sur les comportements à adopter dans le contexte de l'élection présidentielle. Le graffiti en scénettes initié par ces étudiants, sur commande de l'USAID, se caractérise par des dessins faisant preuve d'esthétique tant au niveau de la forme qu'au niveau du fond. Les photos ci-après servent d'illustration de cette technique d'art urbain à Abobo, précisément dans le quartier Sogefiha. 
Illustration 6 - Qui institutionnalise le street art?

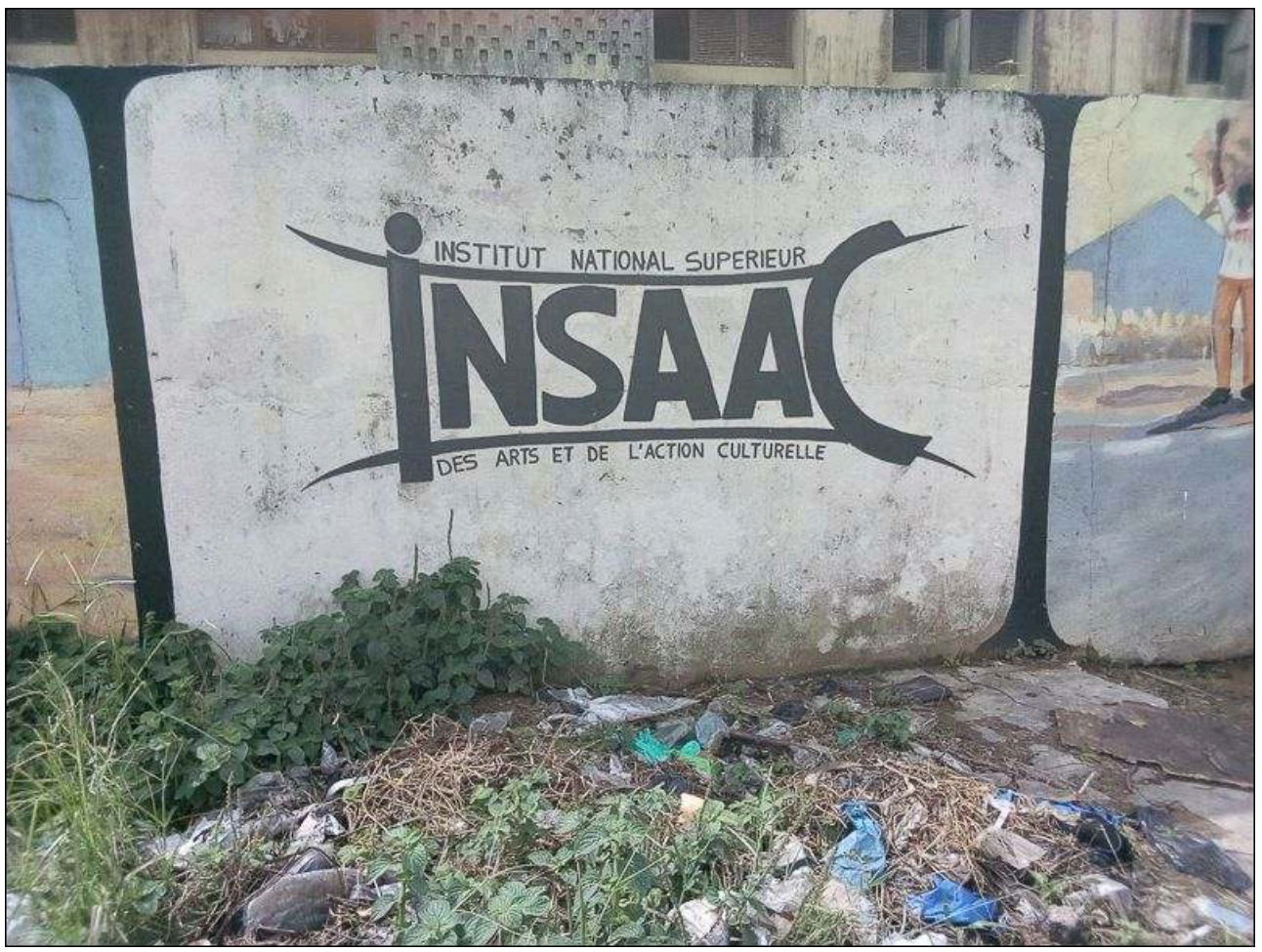

L'Institut National Supérieur des Arts et de l'Action Culturel est celui qui officialise le street art en général et le graffiti en particulier dans la commune d'Abobo durant la campagne électorale en 2010 Auteur : I. Sagnon, 2017 
Illustration 7 - Sensibilisation d'enrôlement en 2010

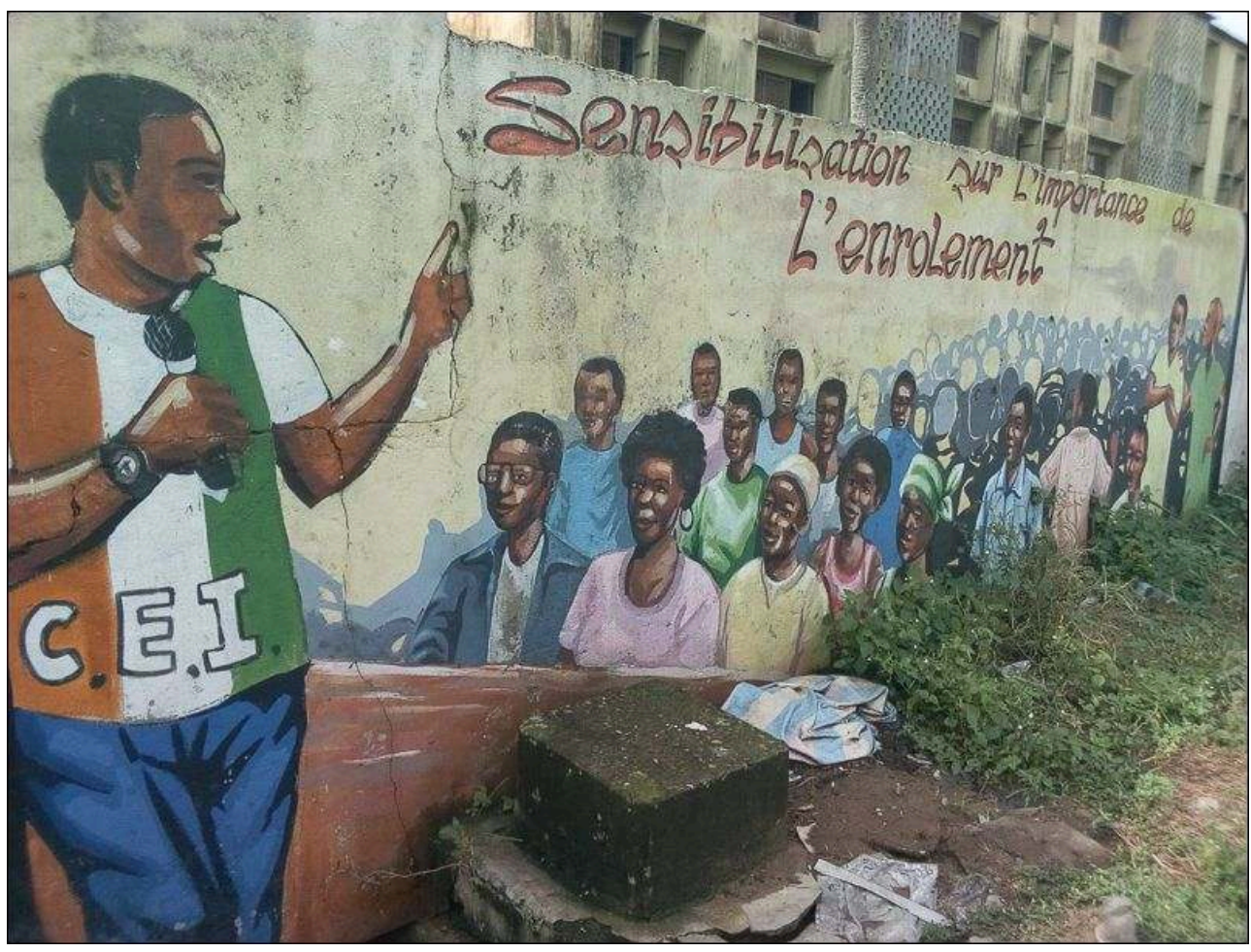

Un agent de la Commission Électorale Indépendante sensibilise des électeurs ivoiriens sur l'importance de l'enrôlement dans le processus de vote de l'élection présidentielle de 2010. Auteur : I. Sagnon, 2017. 
Illustration 8 - Campagne électorale d'un candidat

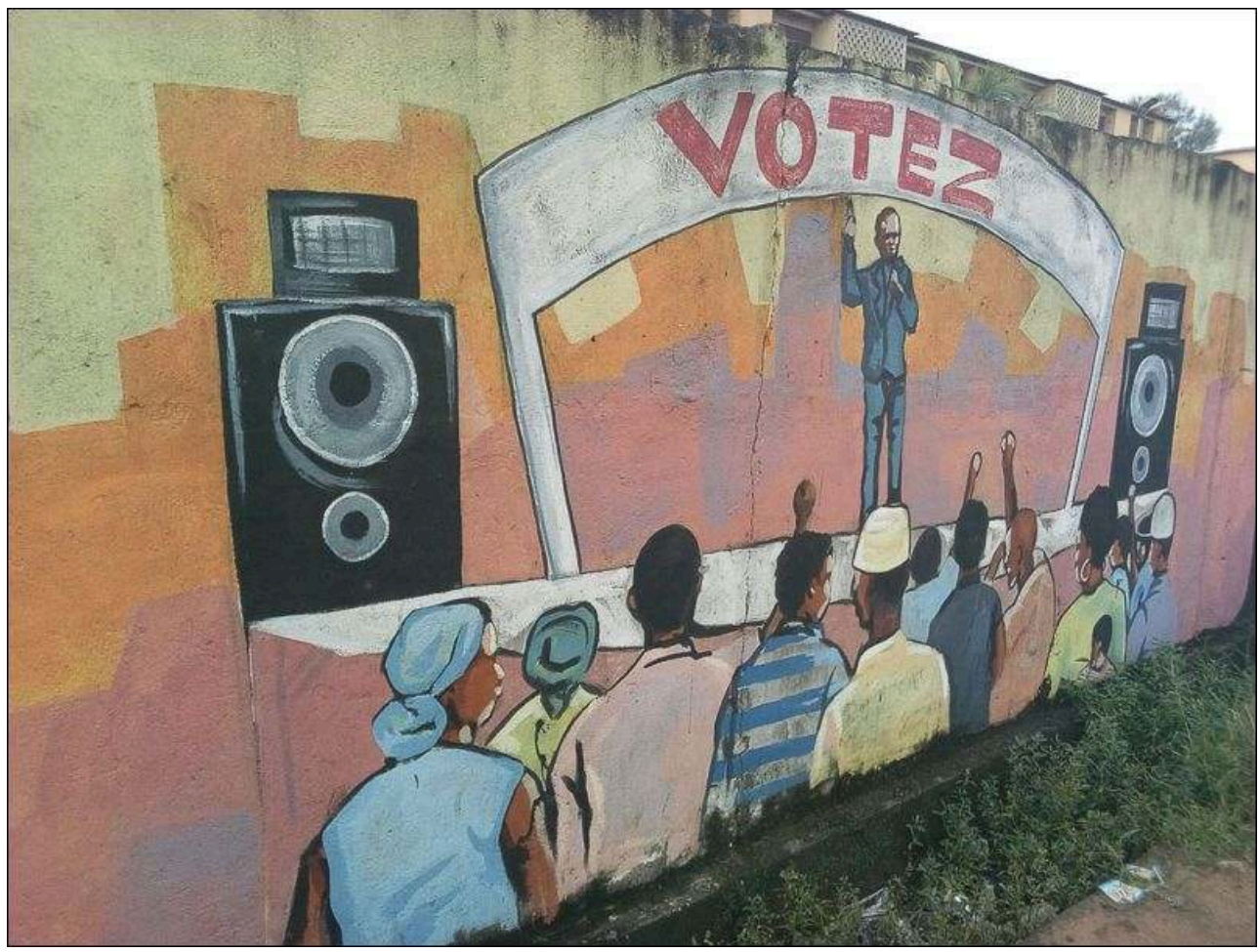

Au travers de cette image, il est demandé à chaque candidat de présenter dans la discipline son programme de gouvernance aux électeurs, dans l'optique de convaincre ces derniers à voter pour lui. Auteur : I. Sagnon, 2017. 


\section{Illustration 9 - Campagne électorale de partisans}

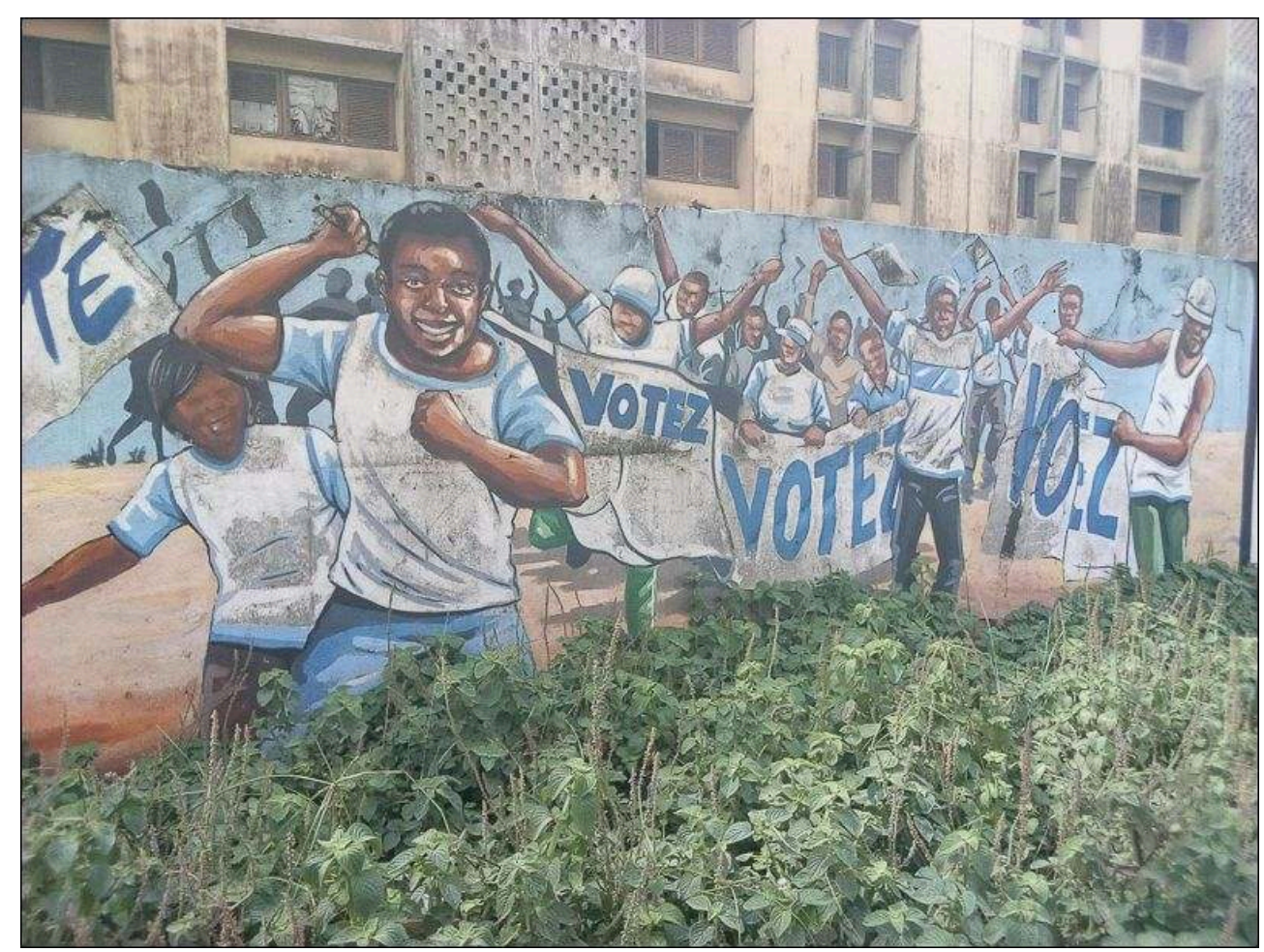

Le déroulement de la campagne électorale de partisans d'une formation politique, qui au regard de l'image devrait être apaisé et dans la joie.

Auteur : I. Sagnon, 2017. 
Illustration 10 - Mobilisation des électeurs

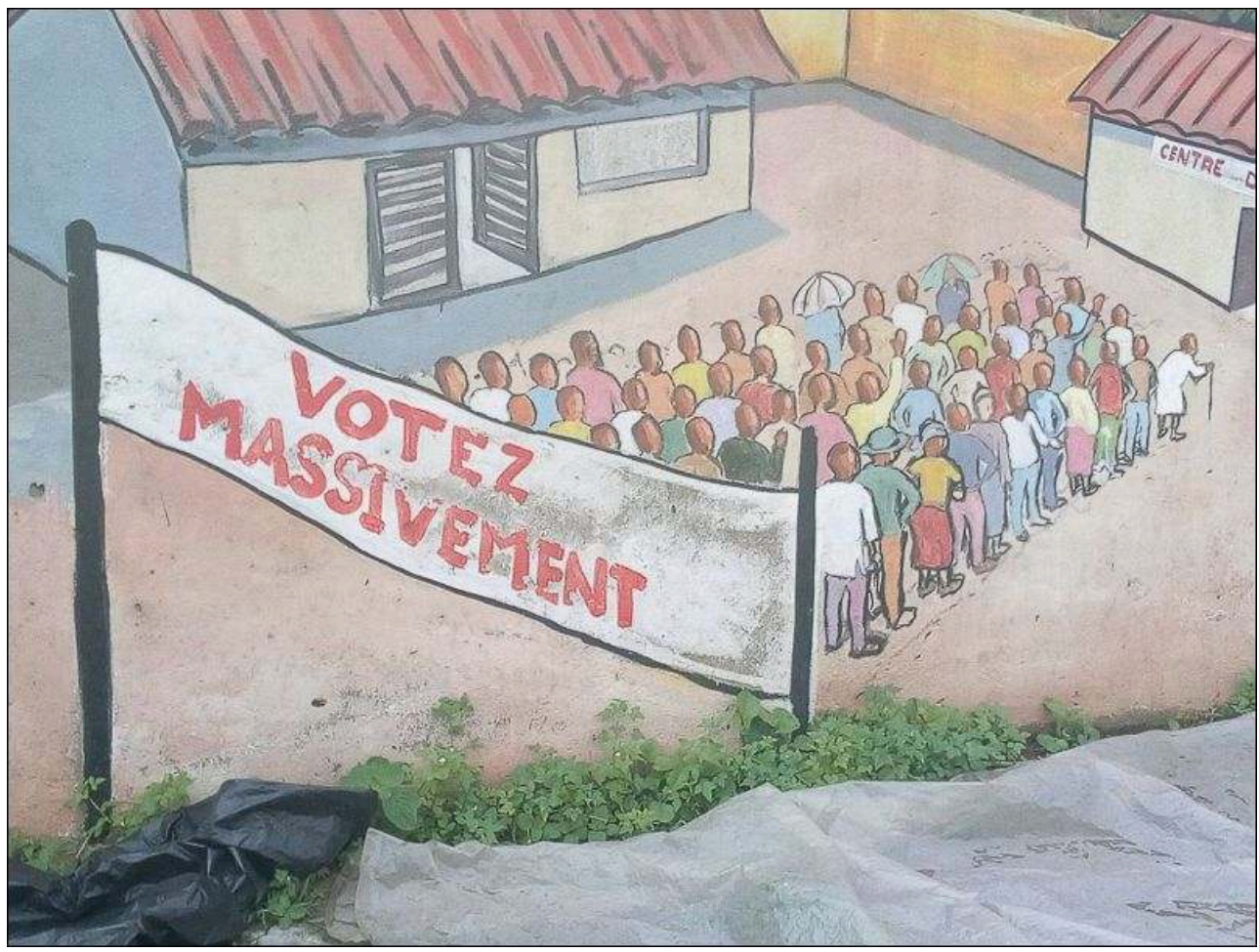

Il est souhaité une forte mobilisation des électeurs le jour de vote et cela dans la discipline. Auteur : I. Sagnon, 2017 
Illustration 11 - Où voter ?

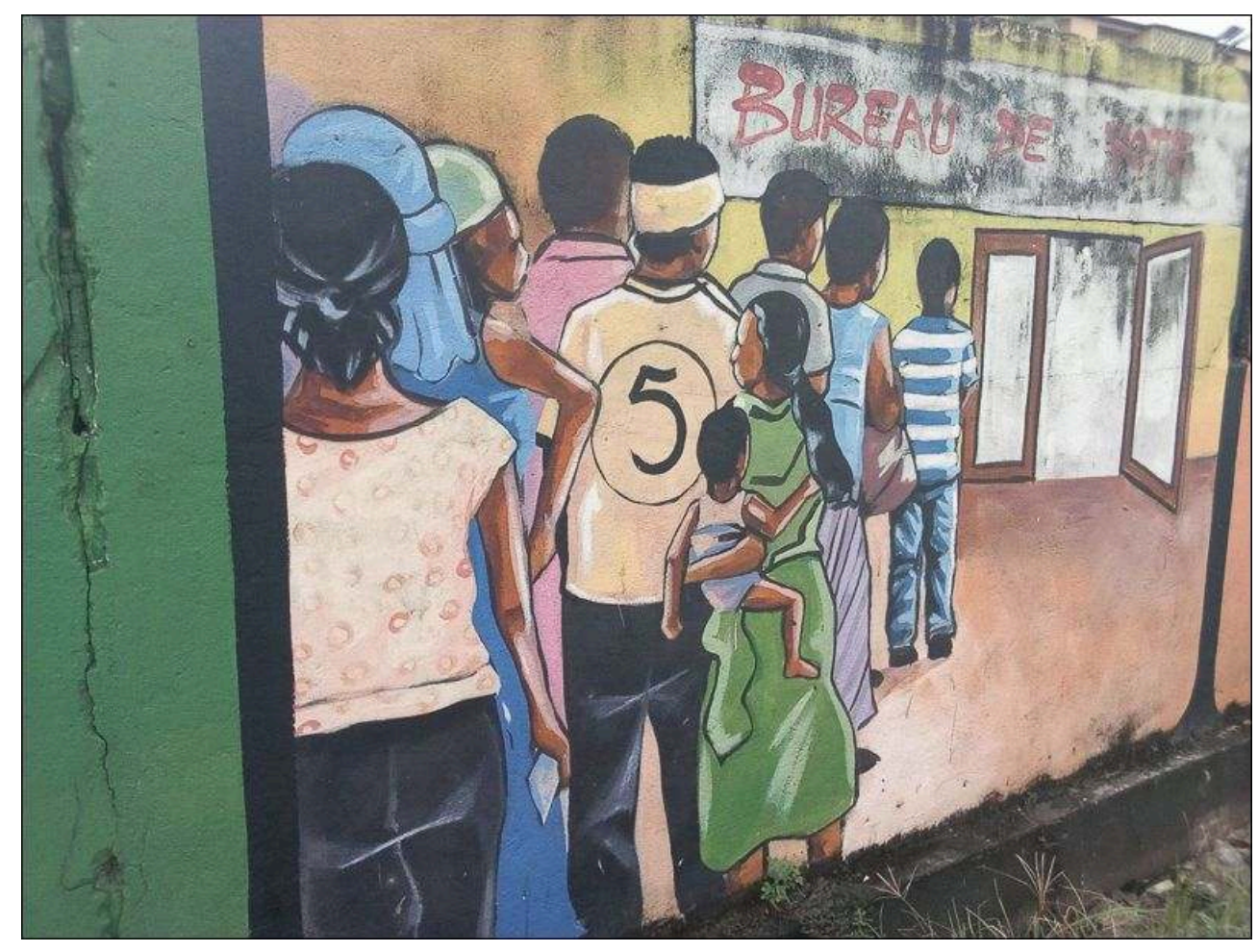

L'image indique que les électeurs doivent se rendre dans la salle indiquée comme bureau de vote afin de choisir leur candidat.

Auteur : I. Sagnon, 2017. 
Illustration 12 - L'isoloir de vote

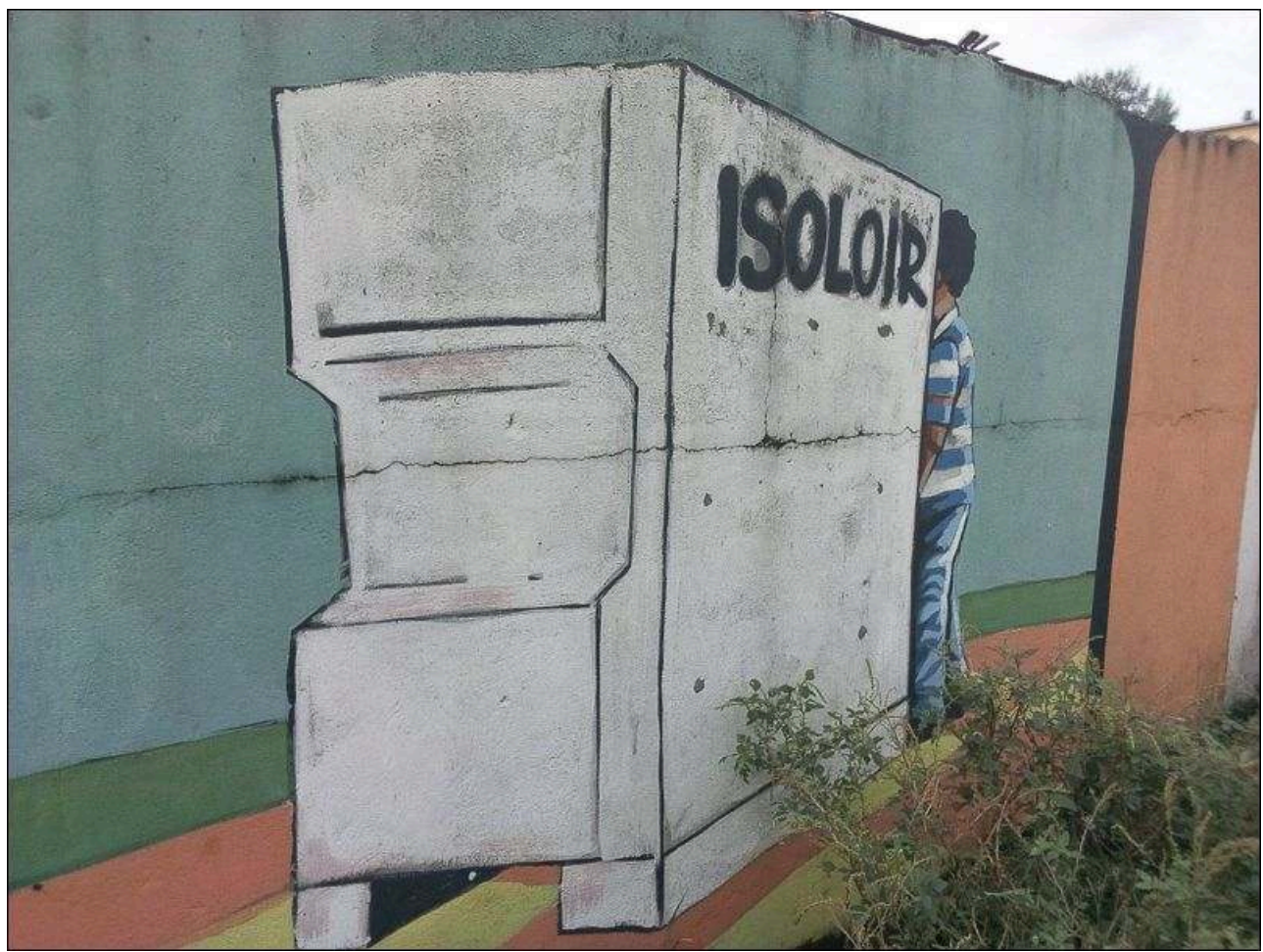

Après avoir récupéré le bulletin de vote auprès des agents mandatés par la C.E.I., il est recommandé à l'électeur de se cacher dans l'isoloir afin d'être l'unique témoin de son suffrage.

Auteur : I. Sagnon, 2017. 
Illustration 13 - Comment finaliser le vote?

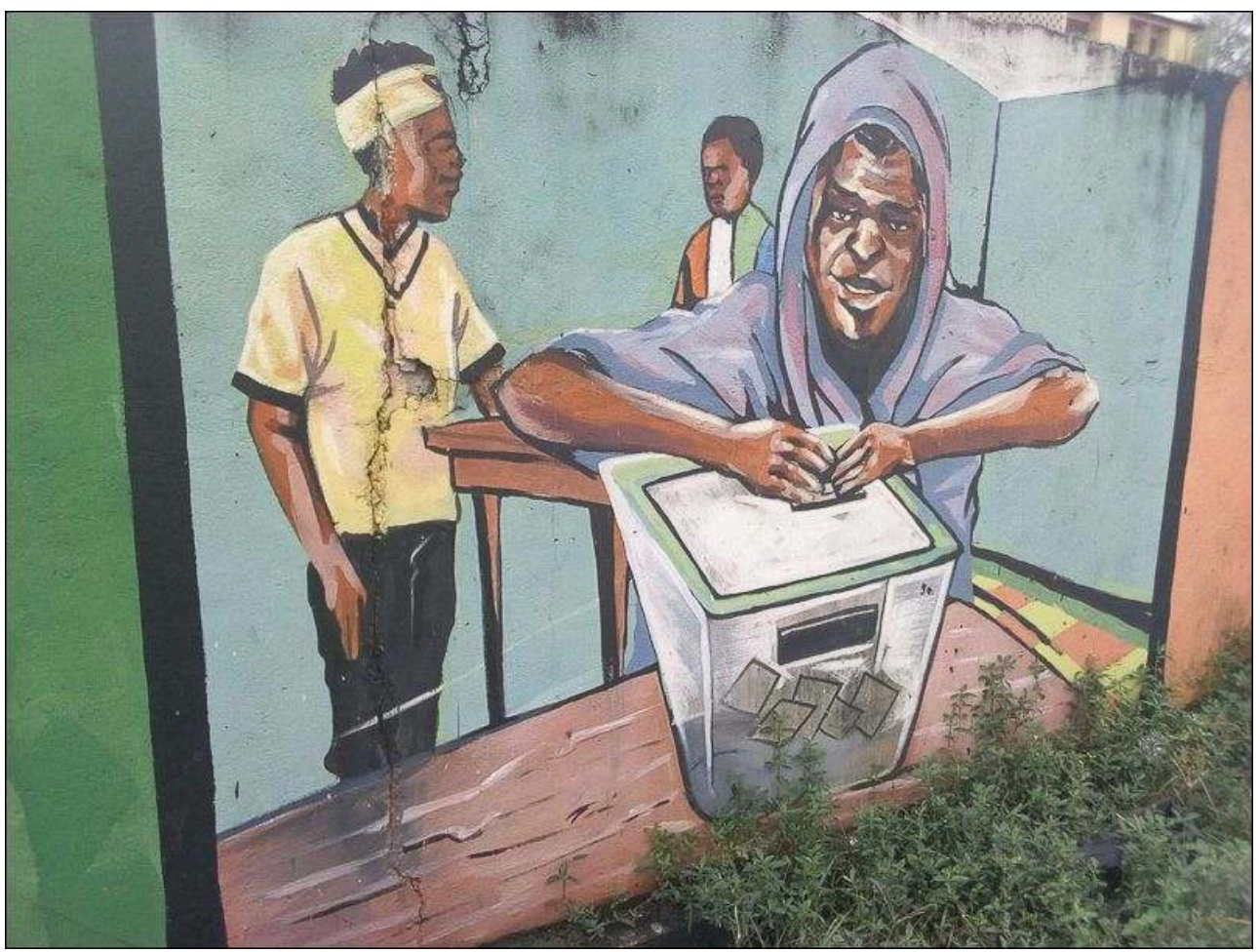

Après la désignation du candidat de son choix dans l'isoloir, l'électeur finalise son vote en mettant le bulletin dans l'urne en présence de représentants des partis politiques et d'agents de la C.E.I.

Auteur : I. Sagnon, 2017. 
Illustration 14 - Proclamation des résultats électoraux

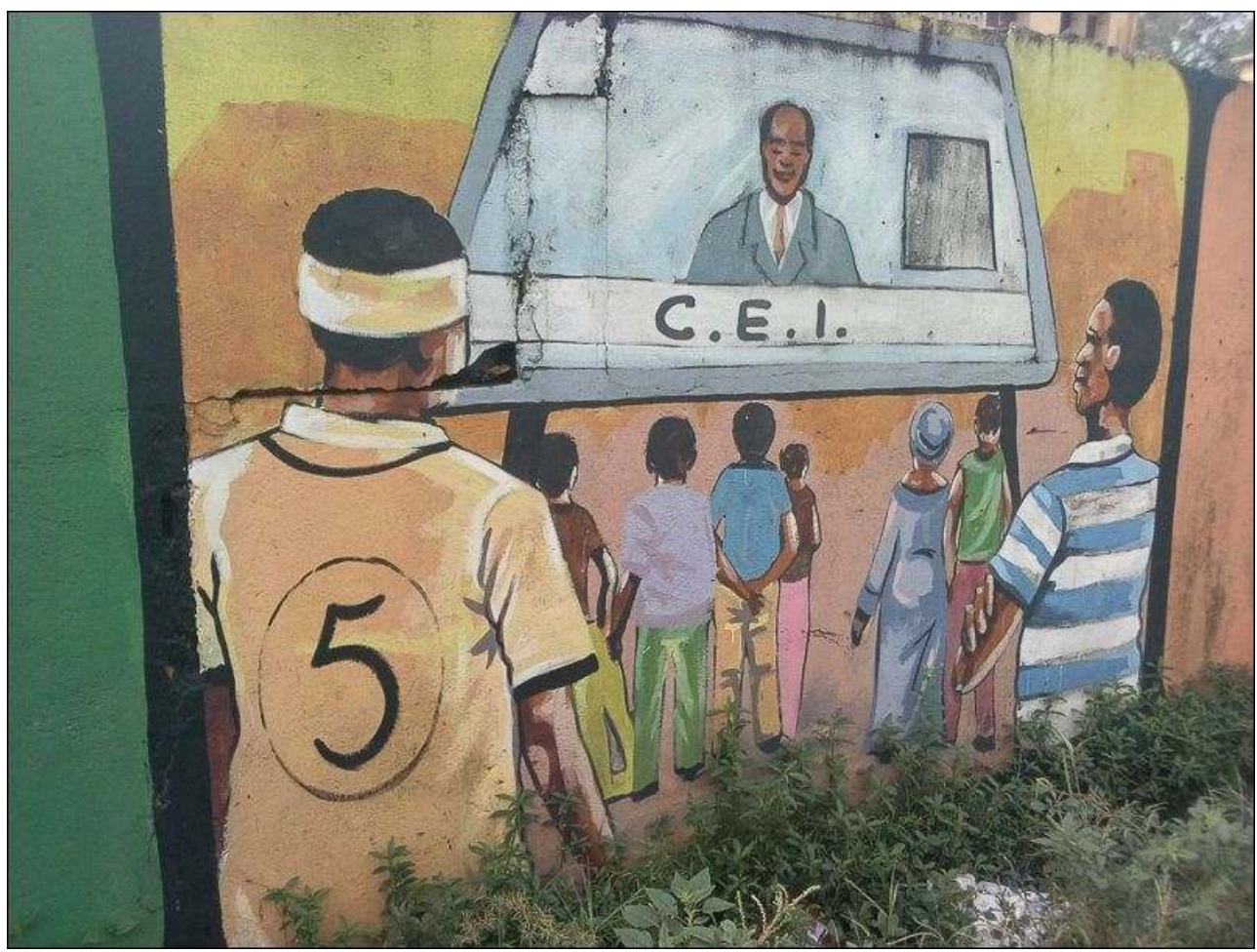

La proclamation des résultats électoraux est du ressort de la C.E.I., qui invite les citoyens à suivre cela en particulier à la télévision.

Auteur : I. Sagnon, 2017. 


\section{Illustration 15 - Une pensée à bannir}

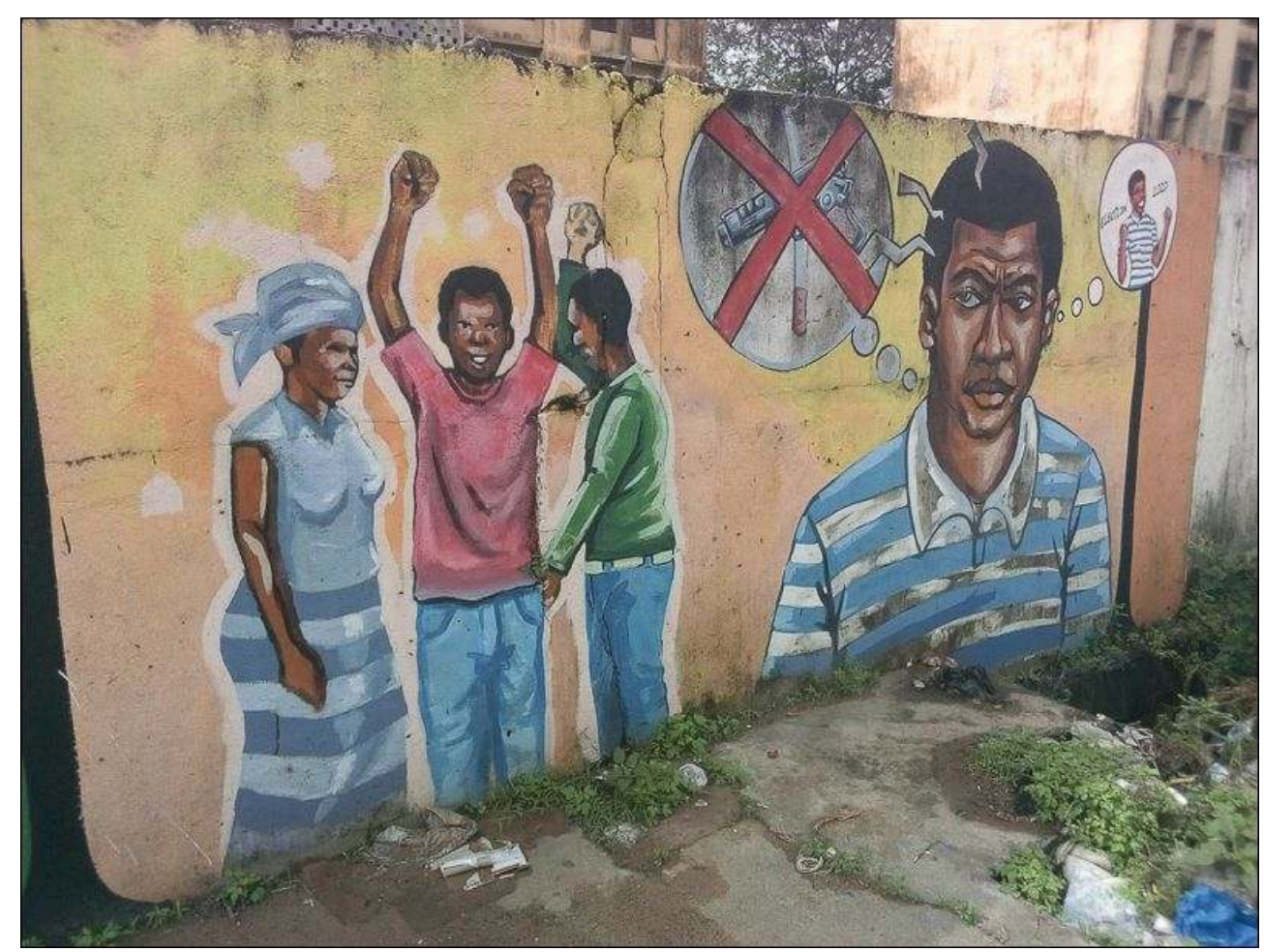

L'électeur dont le candidat a perdu l'élection est exhorté à ne pas recourir aux armes contre des partisans manifestant la victoire de leur candidat.

Auteur: I. Sagnon, 2017. 
Illustration 16 - Congratulation électorale

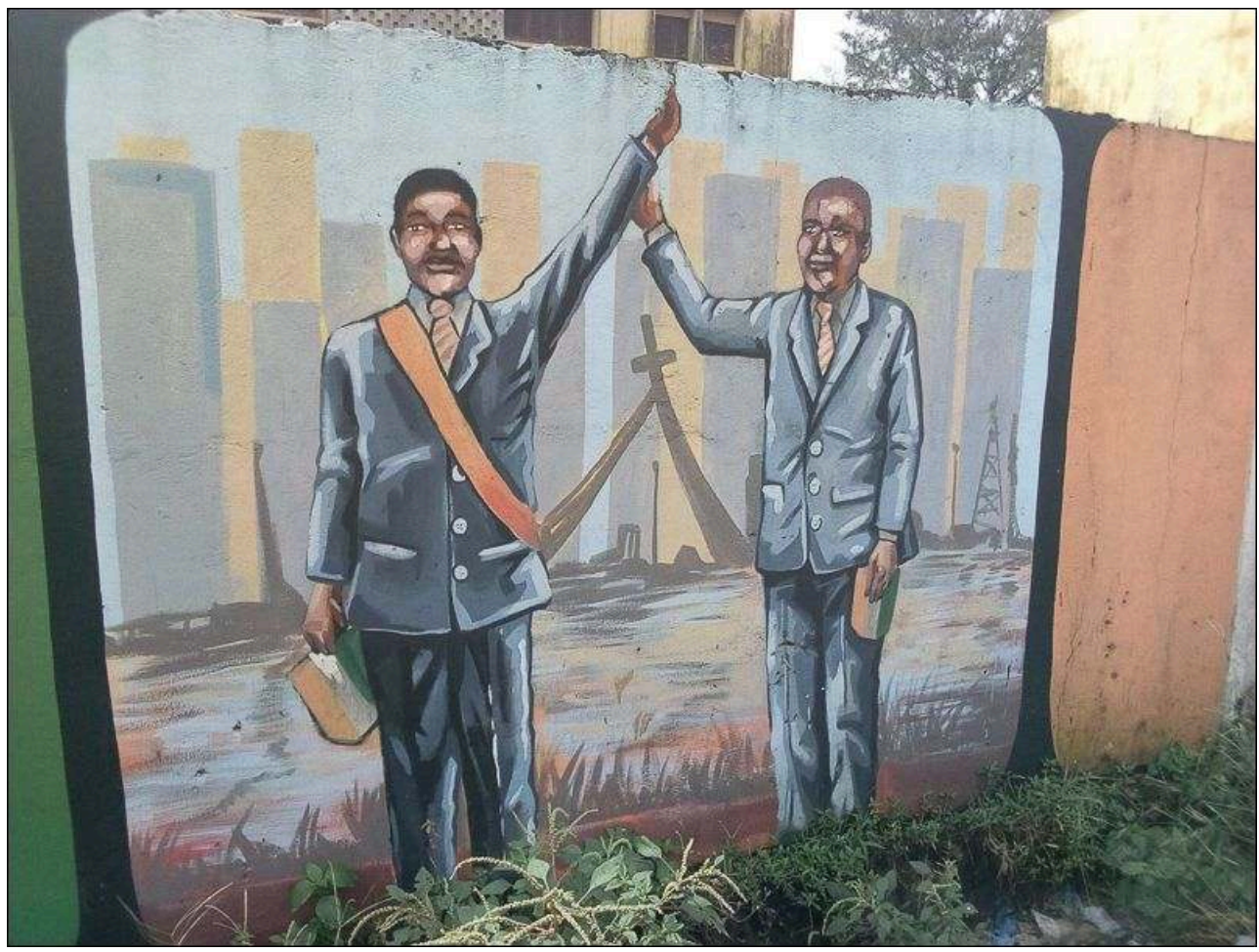

Il est conseillé au candidat perdant d'accepter sa défaite et de féliciter son homologue gagnant. Auteur: I. Sagnon, 2017. 


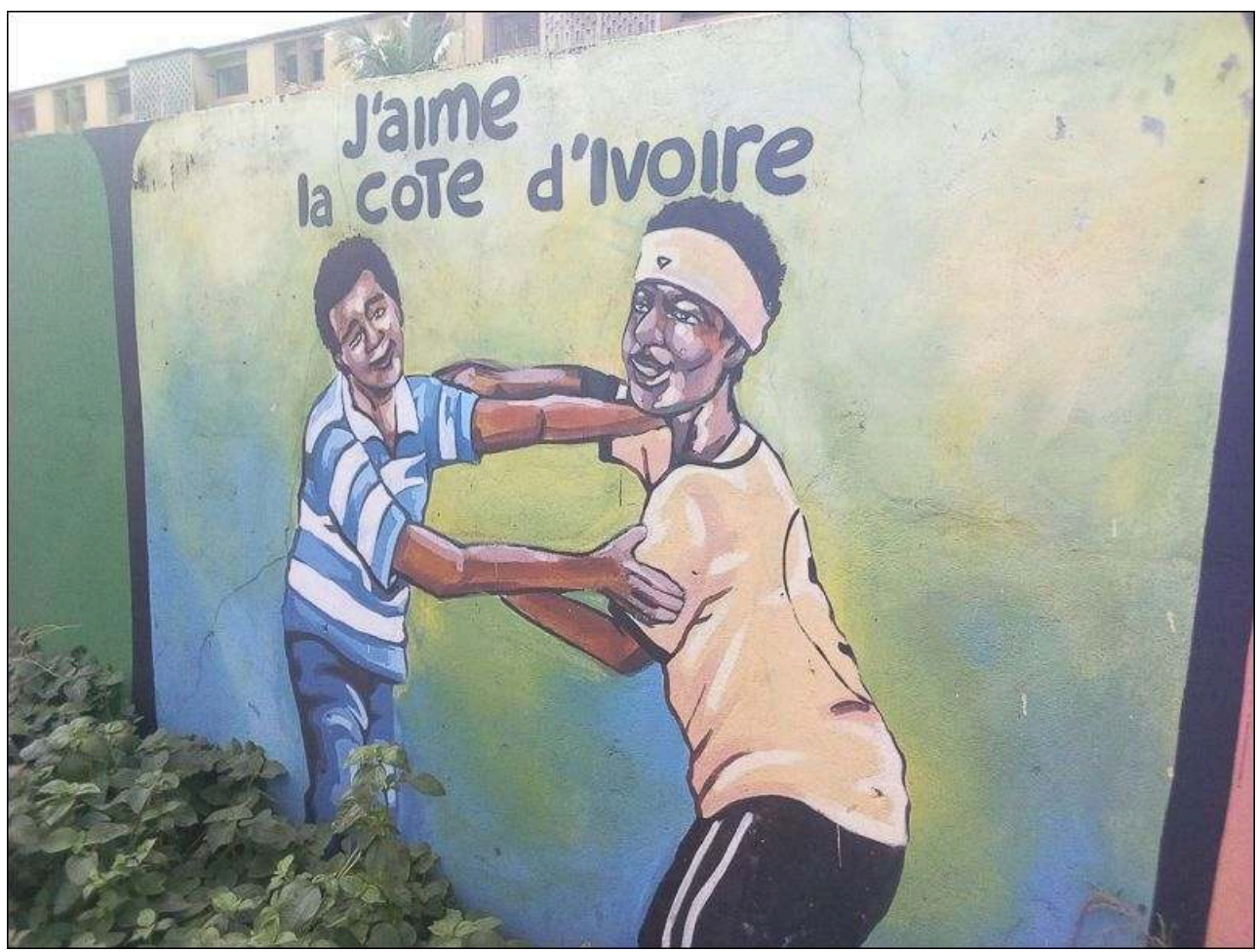

Au-delà du résultat électoral et de l'appartenance à différents partis politiques, il est conseillé aux partisans de s'unir pour un intérêt commun à savoir la Côte d'Ivoire. L'amour pour son pays doit alors animer tous les Ivoiriens sans aucune distinction à œuvrer pour une cohésion nationale.

Auteur : I. Sagnon, 2017

Les illustrations 7 à 17 montrent des scénettes faisant la promotion des comportements à adopter lors de l'élection présidentielle de 2010, l'illustration 6 présentant l'établissement d'où proviennent les auteurs de ces graffitis à savoir l'INSAAC, situé dans la commune de Cocody. L'illustration 7 sensibilise à l'importance de l'inscription sur les listes électorales indispensable pour le vote. Cette sensibilisation se fait par un agent de la Commission Électorale Indépendante (CEI), la seule à organiser les élections en Côte d'Ivoire depuis l'inscription des citoyens sur les listes électorales jusqu'à la proclamation des résultats. Les illustrations 8 et 9 présentent la campagne électorale qui doit pouvoir se tenir sans empêcher ni nuire à autrui. Les illustrations 10 et 11 exhortent les citoyens inscrits à aller dans leur centre et bureau de vote, à la fois massivement et dans la discipline. L'illustration 12 présente l'isoloir où le citoyen doit se tenir pour choisir son candidat tandis que l'illustration 13 présente l'urne dans laquelle il doit mettre le bulletin de vote. L'illustration 14 invite la population à suivre la proclamation des résultats par la seule commission habilitée, à savoir la CEI. L'illustration 15 condamne l'utilisation d'arme à feu et arme blanche en cas de défaite électorale tandis que l'illustration 16 exhorte le candidat perdant à congratuler son homologue gagnant. Pour terminer, l'illustration 17 promeut l'union quel que soit le candidat élu, cela dans l'intérêt du pays qui est le bien commun de tous les Ivoiriens.

Ce graffiti en scénettes visant à éviter une éventuelle crise post-électorale et à retrouver la cohésion nationale perdue depuis 1999 en éduquant aux bons comportements avant, pendant et après l'élection, est réalisé sur le mur de la cité estudiantine, cité qui abrite des étudiants appartenant à la FESCI constituant la 
population cible de cette campagne. La cité était en effet pour ces derniers, le lieu de résidence, de rassemblement, de prise de décisions en un mot leur fief où des propos tels que "Vous allez voir dans ce pays, on va vous mater " pouvaient être clamés par ces «fescites » à l'encontre des partisans du parti de l'opposition (RDR). Le mur de la cité universitaire est alors jugé idéal par l'USAID, via les étudiants de l'INSAAC, pour servir de support au graffiti en scénettes. De plus, la cité estudiantine se trouve face à une gare de bus de la SOTRA (Société des Transport Abidjanais), regroupant une dizaine de lignes reliant Abobo aux autres communes d'Abidjan et étant, dès lors, particulièrement sollicitée (en moyenne 1500 passagers par jour). Ces voyageurs représentent également une population cible pour le commanditaire de la campagne de sensibilisation. Les passagers de la SOTRA devaient contribuer à la diffusion des messages de sensibilisation à l'échelle de toute la métropole abidjanaise justifiant l'appropriation du mur de la cité universitaire par cette forme d'art urbain. Interrogé sur ce graffiti, le « fesciste » responsable des chambres en cité s'exprime par personne interposée (2010) en disant ceci :

«Je souligne la beauté des dessins. Ils embellissent la cité universitaire.

Relativement aux messages qu'ils véhiculent, cela n'est pas nouveau. Au-delà de ces

dessins, je ne pourrais me prononcer sur le déroulement et l'après élection car nous

sommes des millions à être concernés ».

13 Ces propos résument également l'avis des piétons et passagers interrogés sur les lieux. Ils apprécient cette technique en ce sens qu'elle enseigne, éduque, sensibilise et soumet de beaux dessins, agréables à admirer. Mais, au-delà de la valorisation territoriale qu'offre ce graffiti en pièces, l'objectif escompté n'a pas été atteint. En effet, suite à l'élection présidentielle de 2010, la Côte d'Ivoire a à nouveau plongé dans une crise socio-politique et militaire occasionnant d'importants dégâts matériels et des pertes en vie humaine particulièrement dans la commune d'Abobo. Le premier magistrat de la commune ne reste pas indifférent. Soutenu par les six députés de ladite commune (partisans du RDR et du PDCI), le maire initie durant les premières années d'accalmie des installations lumineuses en hommage aux martyres de la crise post-électorale.

\section{L'installation lumineuse : un outil d'hommage aux martyrs en 2012}

14 Après avoir conquis les murs de certains bâtiments publics, élèves et étudiants sont rejoints dans cette culture d'art urbain par la commune d'Abobo. En effet, l'installation lumineuse va constituer pour la municipalité un outil d'hommage et de compassion à l'endroit de la population communale en général et des partisans du RDR en particulier, formation politique dont le maire est membre et qui remporte l'élection présidentielle de 2010. Au regard de cette affinité politique et du sacrifice de la vie de militants de ce parti, le maire d'Abobo met en place des installations lumineuses durant les deux dernières semaines de l'année 2012. Cette initiative municipale perdure jusqu'en 2014. Les illustrations ci-après permettent d'appréhender les représentations cognitives de la municipalité à travers l'installation lumineuse de 2012 et 2014. 
Illustration 18 - Hommage aux martyrs en 2012

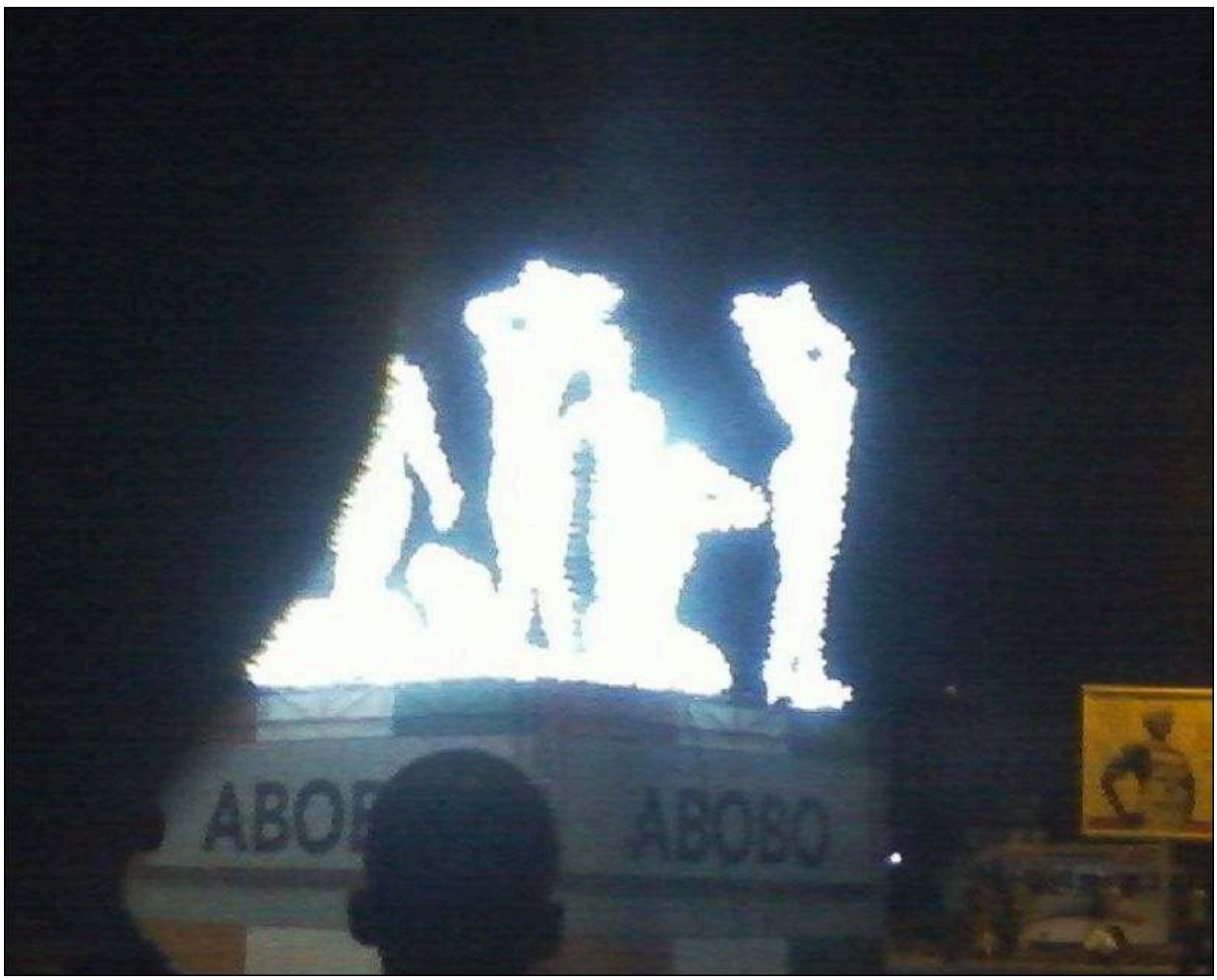

Un hommage à travers l'installation lumineuse est rendu aux partisans du RDR morts durant la crise post-électorale de 2010. Cette forme d'art urbain fut l'œuvre de la municipalité d'Abobo dont les membres appartiennent au même parti politique.

Auteur : I. Sagnon, 2012 
Illustration 19 - La colombe, expression d'amour et de compassion

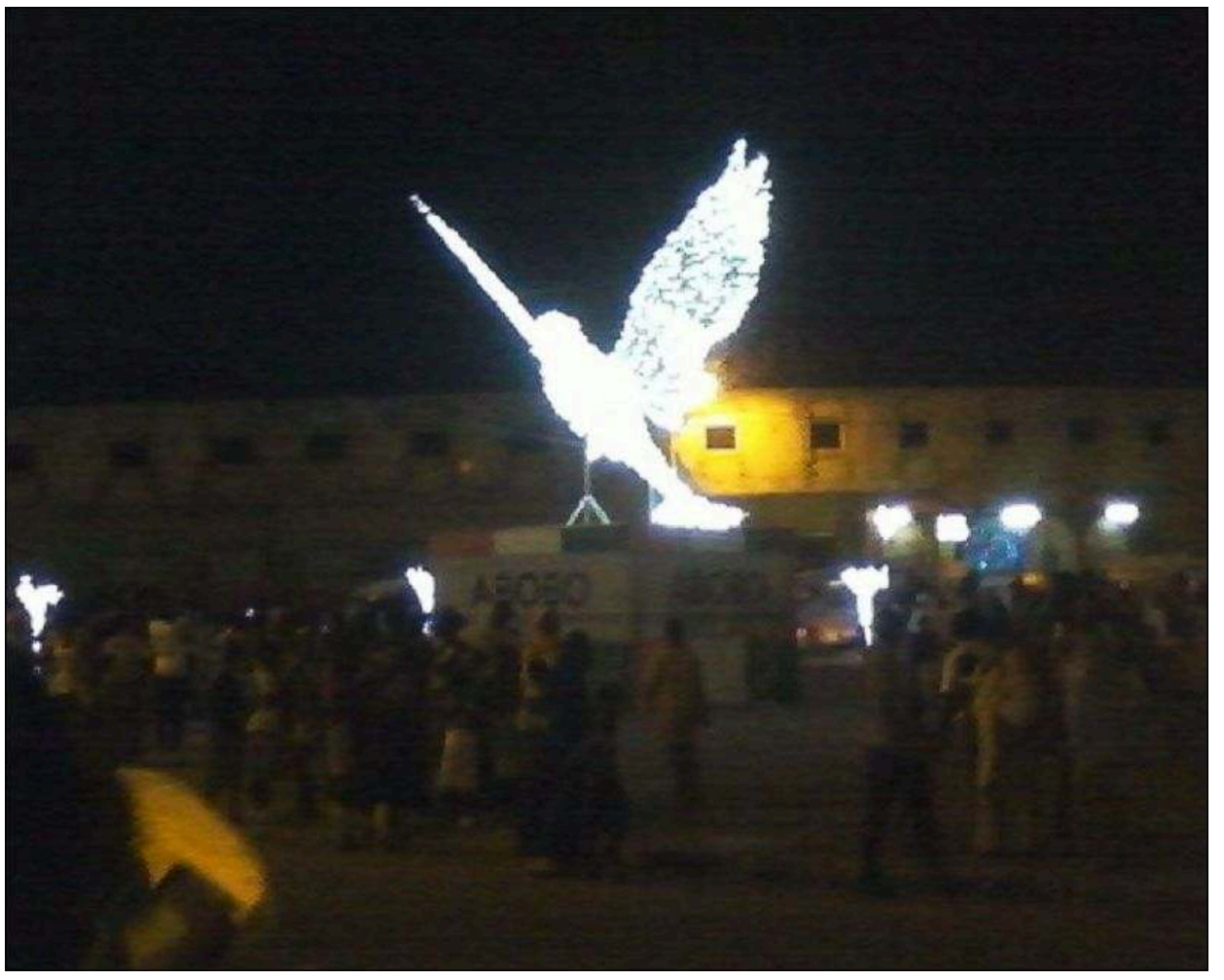

À travers la blancheur de la colombe, la municipalité d'Abobo exprime sa compassion aux familles endeuillées.

Auteur: I. Sagnon, 2012. 
Illustration 20 - L'armoirie, une marque de reconnaissance nationale

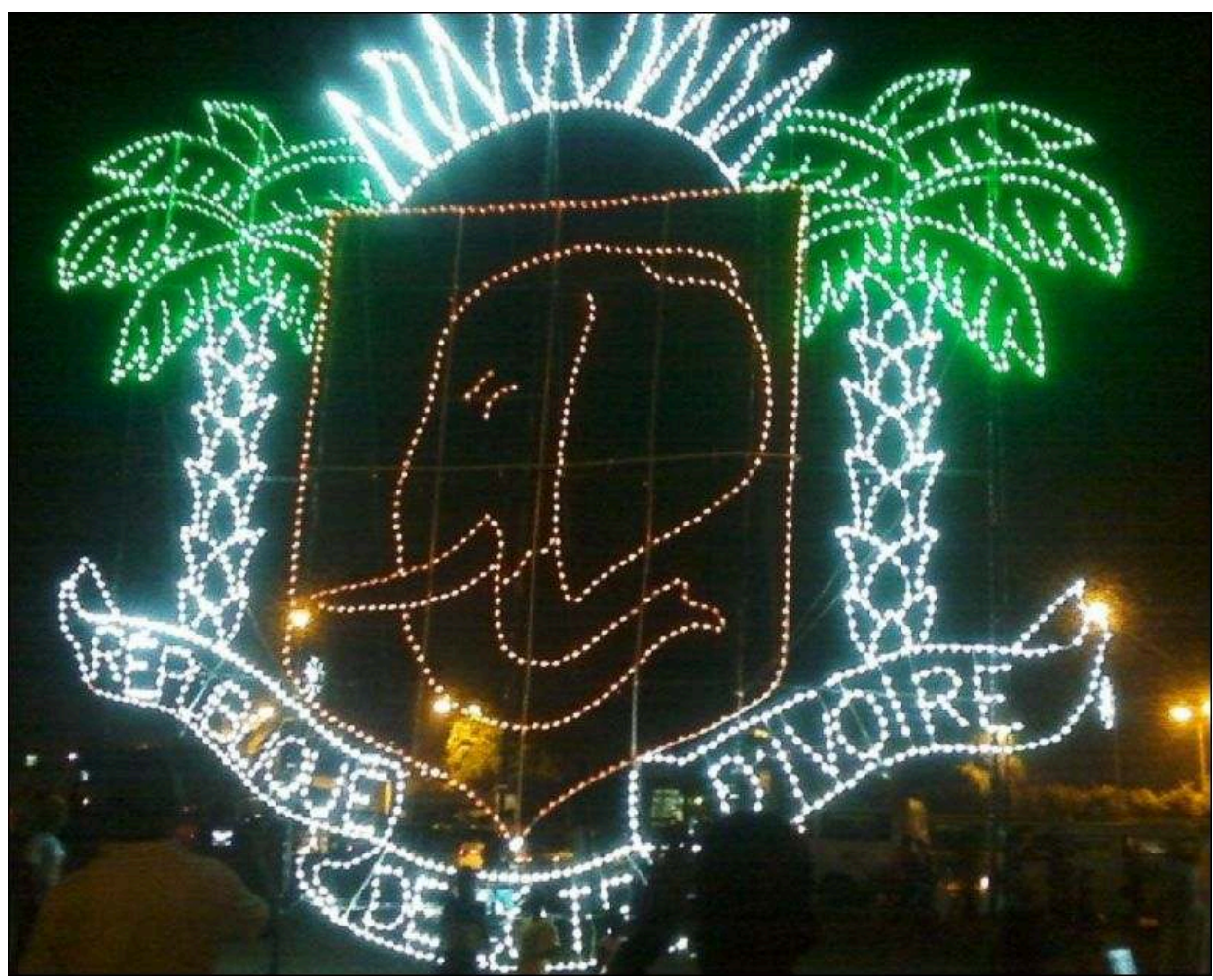

Par le canal de l'armoirie de la Côte d'Ivoire, le candidat victorieux du RDR à l'élection présidentielle de 2010 exprime sa gratitude au regard des efforts consentis par ses partisans. À travers cette installation lumineuse, il déplore toutefois les pertes en vie humaine au prix de la stabilité sociopolitique.

Auteur : I. Sagnon, 2014 
Illustration 21 - Le rappel à l'ordre du gouvernement ivoirien

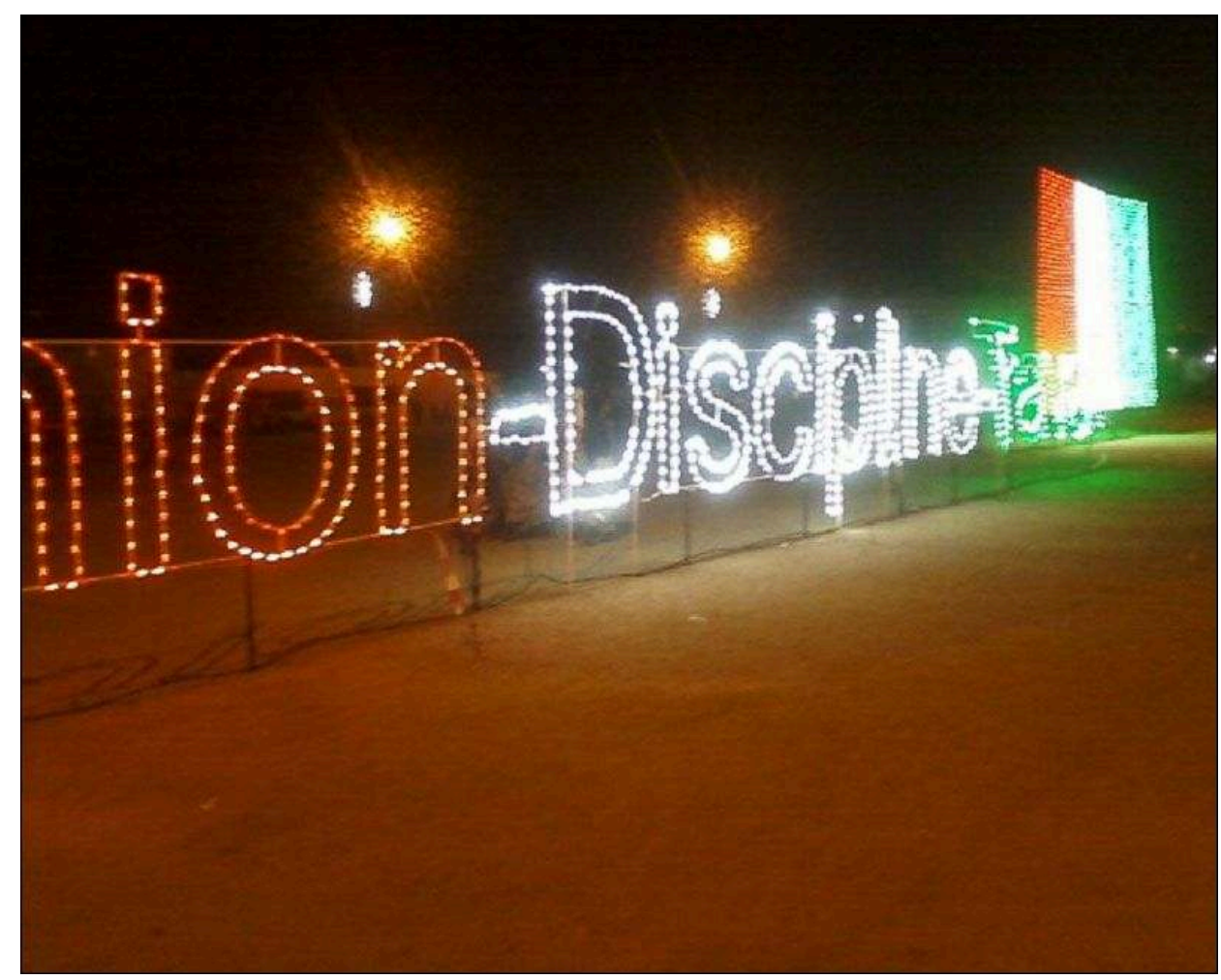

La devise de la république de Côte d'Ivoire matérialisée par l'installation lumineuse est un message du gouvernement aux Ivoiriens afin de maintenir ensemble la stabilité politique et de travailler dans la concorde pour le développement du pays.

Auteur : I. Sagnon, 2014

15 L'illustration 18 montre des représentations humaines lumineuses, couchées pour certaines et débout pour d'autres, évoquant les familles endeuillées près des corps sans vie. De fait, lors de la crise post-électorale de 2010, des partisans du RDR réclamant le départ de Laurent Gbagbo ont été tués au rond-point du Banco et c'est bien pour leur rendre hommage et exprimer sa compassion que le maire d'Abobo fait réaliser cette œuvre. L'illustration 19 prise également dans le quartier Banco, présente un oiseau de couleur blanche symbolisant l'amour et l'affection politique que le maire avait pour ses partisanes défuntes. Selon l'un de ses adjoints, cet oiseau représente l'ange du paradis guidant les martyres politiques. Les illustrations 20 et 21 présentent respectivement l'armoirie, la devise et l'emblème de la de Côte d'Ivoire. Situées au rond-point d'Abobocentre, en face de la mairie, ces installations lumineuses manifestent l'engagement patriotique du gouvernement ivoirien. C'est un hommage que l'actuel président rend aux femmes martyres d'Abobo en particulier et à tous ceux qui ont contribué à rétablir l'ordre socio-politique dans le pays. De façon spécifique, il utilise la devise de la république pour rappeler tous les Ivoiriens à l'ordre dans l'optique de retrouver la paix, gage de tout développement d'un pays.

16 Concernant la stratégie de localisation des installations lumineuses, soulignons l'appropriation des trottoirs de certains ronds-points de la commune d'Abobo. Ces ronds-points sont, pour la municipalité, des lieux préférentiels de vulgarisation de ces messages. Le choix de ces lieux d'exposition est guidé par l'idée de capter l'attention d'un plus grand nombre de personnes. L'image satellitaire (Landsat) ci-après localise les 
ronds-points ayant abrité les installations lumineuses de 2012 ainsi que celles des autres années.

Illustration 22 - Les ronds-points d'installations lumineuses

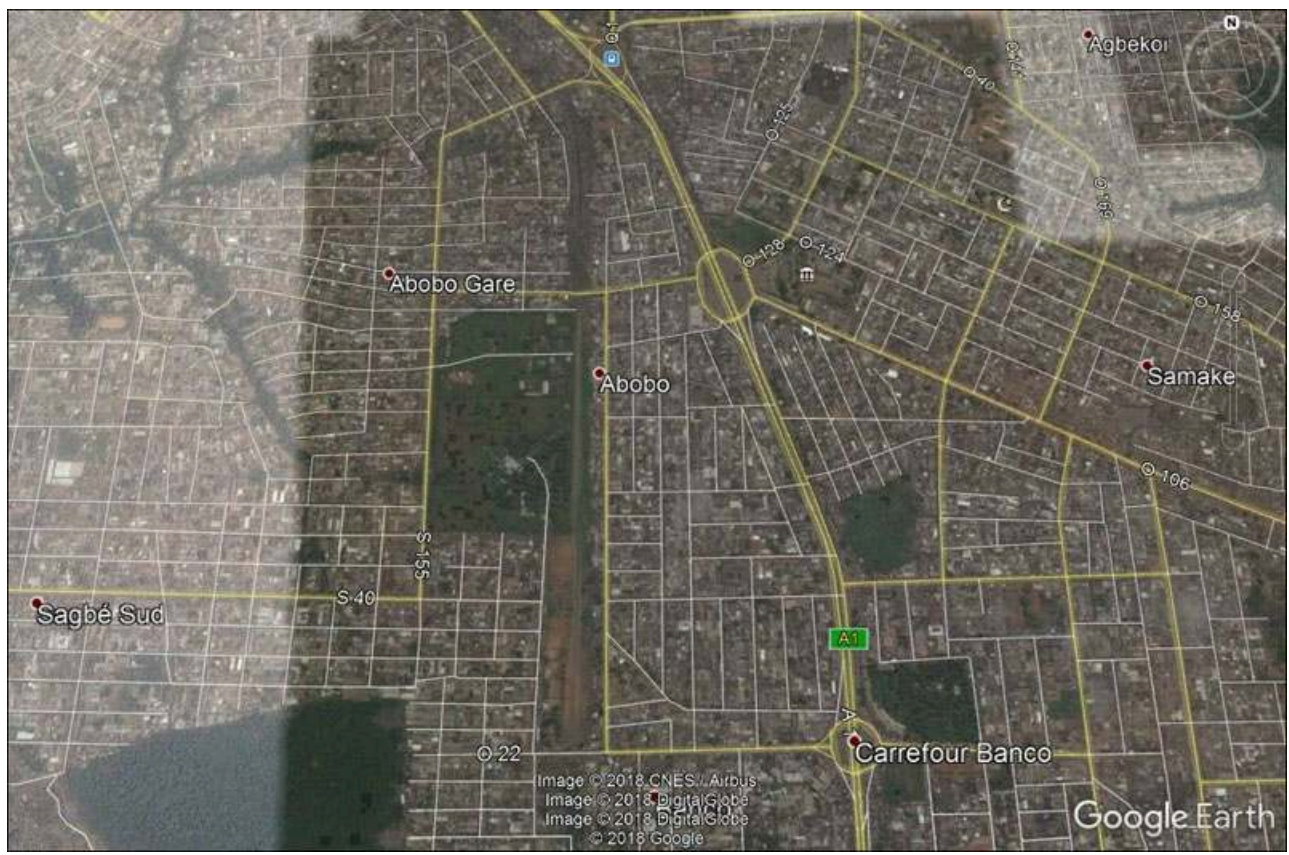

Une localisation stratégique du street art mise en place par la municipalité d'Abobo dans l'optique de capter l'attention et de se faire entendre par un plus grand nombre possible de personnes en 2012, 2013 et 2014.

Source : Google Earth, 2018 ; auteur : I. Sagnon.

17 Trois ronds-points (cercles en jaune) situés au centre de la commune d'Abobo ont abrité les installations lumineuses initiées par le maire. Du bas vers le haut de l'image satellitaire, on aperçoit ces ronds-points avec des routes en traits de couleur jaune. Le rond-point du Banco est le premier en provenance de la commune d'Adjamé. Il permet d'accéder aux quartiers Sagbé, Samaké, Aboboté. Le quartier Abobo-centre, comme son nom l'indique, se situe bien plus au centre de la commune d'Abobo et comprend à lui seul deux ronds-points dont le plus grand de la commune. À proximité de celui-ci, sont installés le grand marché quotidien et les différentes gares routières de transport en commun. Ainsi, ce rond-point est connu de tous les riverains et enregistre des milliers de passants par jour. Pour la mairie d'Abobo qui y trouve son édifice, c'est l'un des lieux préférentiels pour capter l'attention des populations et véhiculer ses messages. Distant d'une trentaine de mètres de la brigade de gendarmerie d'Abobo, le second rond-point $\mathrm{du}$ quartier (tout au-dessus) a aussi fait l'objet d'installations lumineuses. Il relie les quartiers de Céleste (à l'Ouest), d'Abobo Avocatier, PK 18 (au Nord) et Sogefiha, Plaque (à l'Est). En raison de leur rôle de desserte de nombreux quartiers densément peuplés de la commune, ces ronds-points sont très fréquentés, tant par des automobilistes que par des piétons. Les trottoirs de ces ronds-points, y compris la route principale A1 reliant Adjamé à Anyama tout en traversant la commune d'Abobo, ont donc été choisis par la municipalité à dessein. Profitant des congés de Noël pour en faire bénéficier aussi les élèves et étudiants (toute la jeunesse d'Abobo), les installations lumineuses ont été mises en place en décembre 2012, 2013 et 2014. Durant ces trois premières années de 
stabilité socio-politique, les espaces publics faisant l'objet d'installations lumineuses ont été envahis par la population durant des nuits ${ }^{3}$.

Illustration 23 - Installation lumineuse unicolore

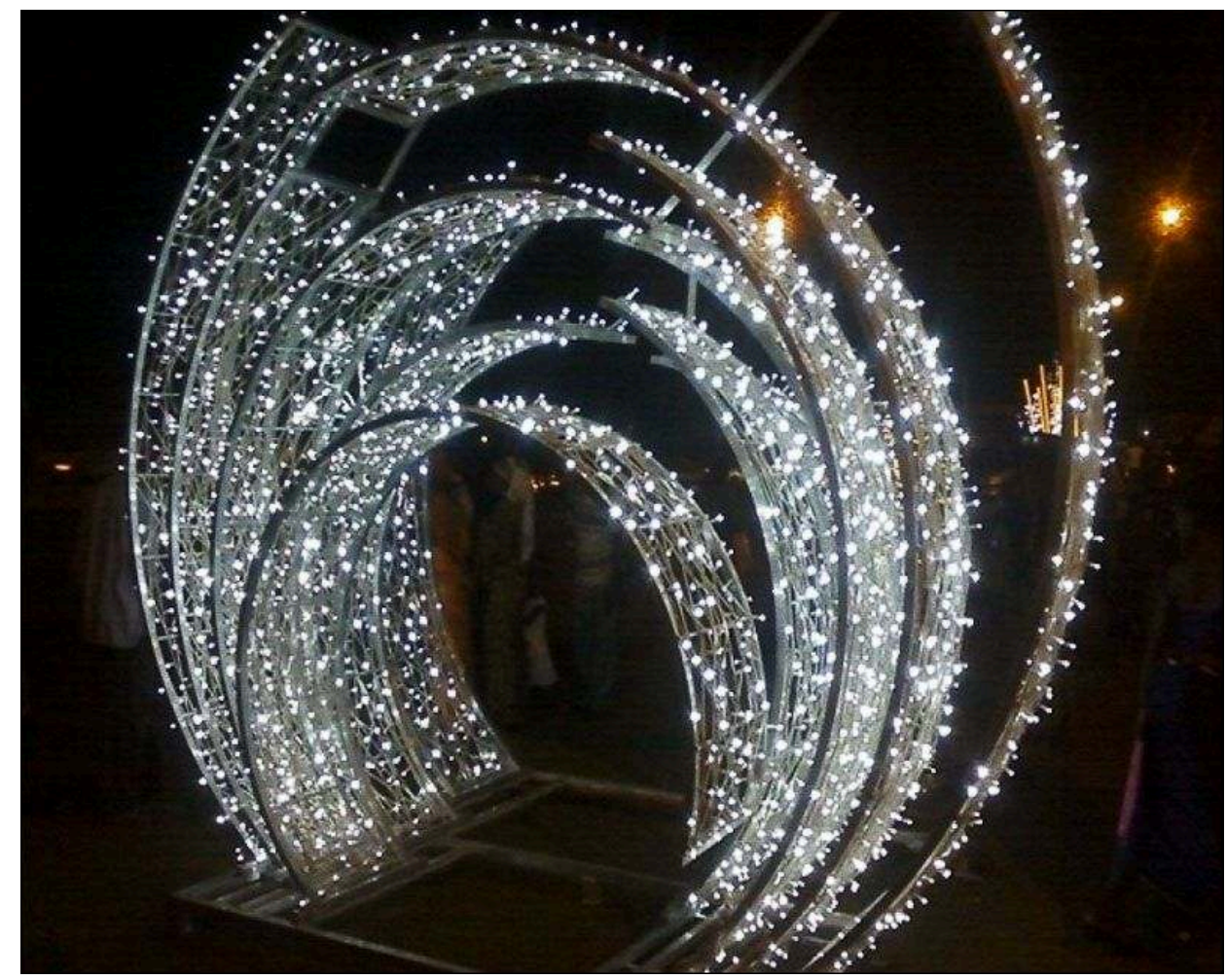

Une installation lumineuse de couleur blanche, de forme arrondie au bonheur des enfants. Auteur : I. Sagnon, 2012 
Illustration 24 - Une attractivité par la lumière

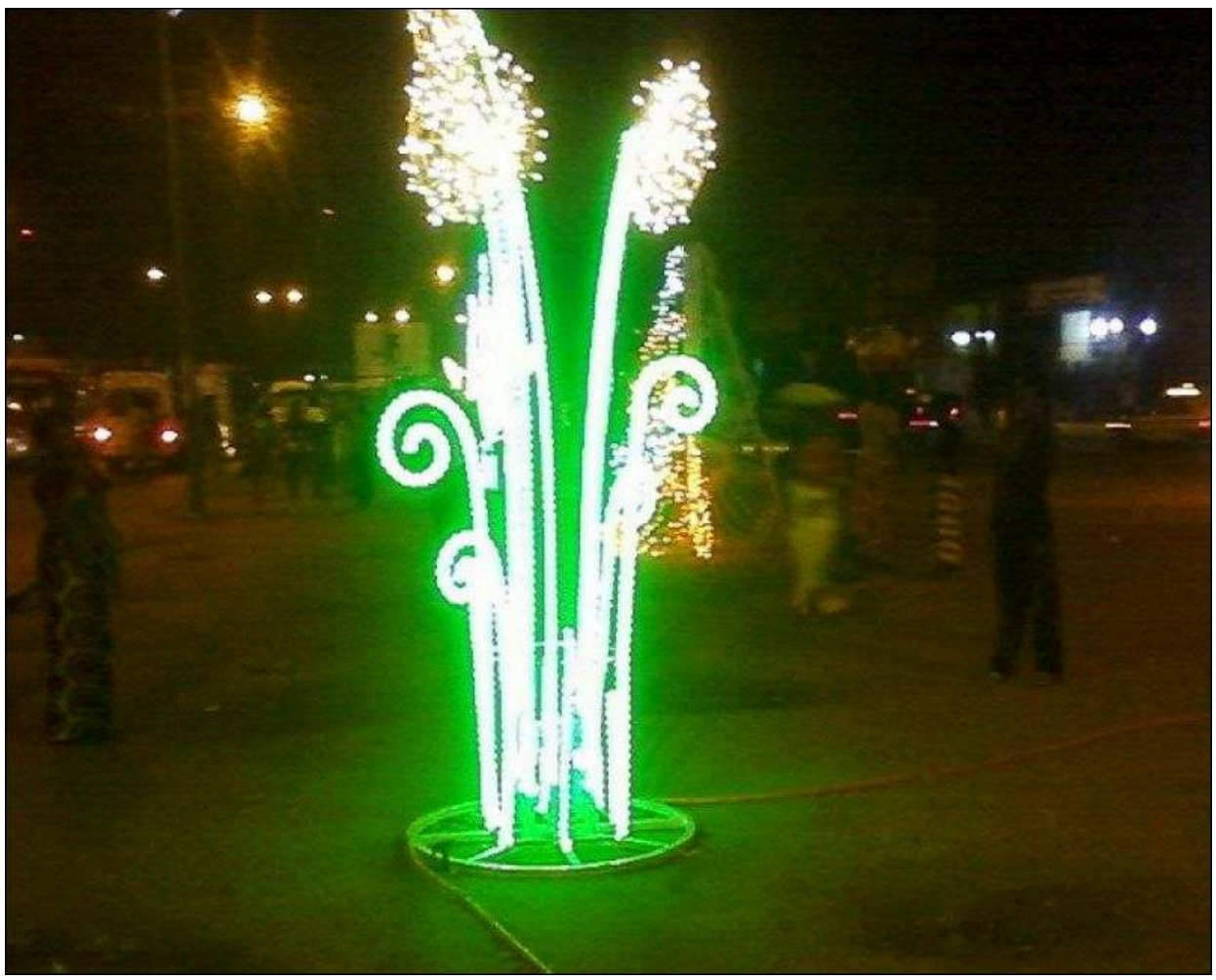

Une couleur verte prédominante illumine un ensemble de barres de fer multiformes au rond-point de la mairie d'Abobo. Cette installation lumineuse favorise l'attractivité territoriale.

Auteur : I. Sagnon, 2012. 
Illustration 25 - Installation lumineuse multicolore

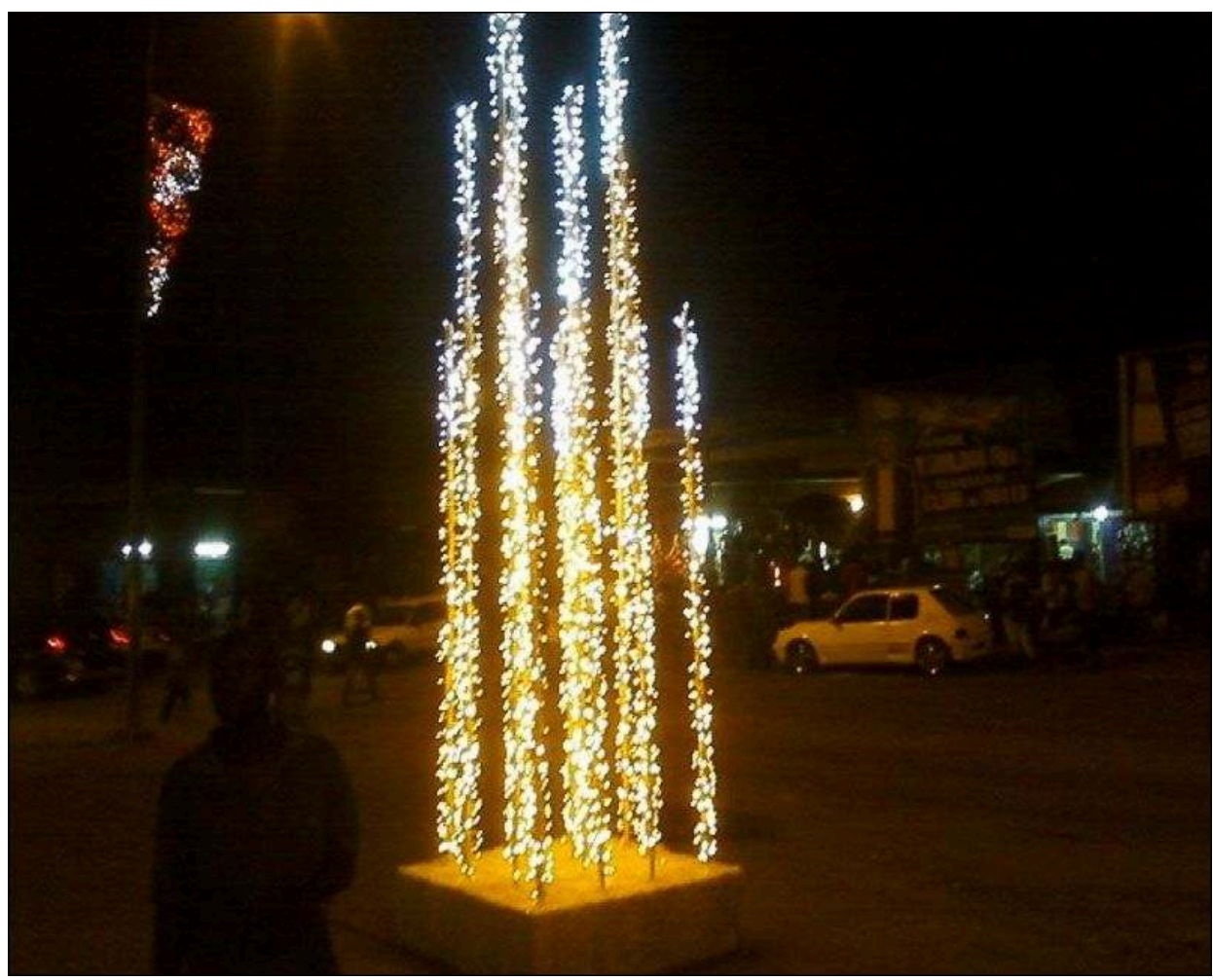

La combinaison de couleurs caractérise certaines installations lumineuses. Cette représentation matérialise une fontaine d'eau.

Auteur : I. Sagnon, 2012 


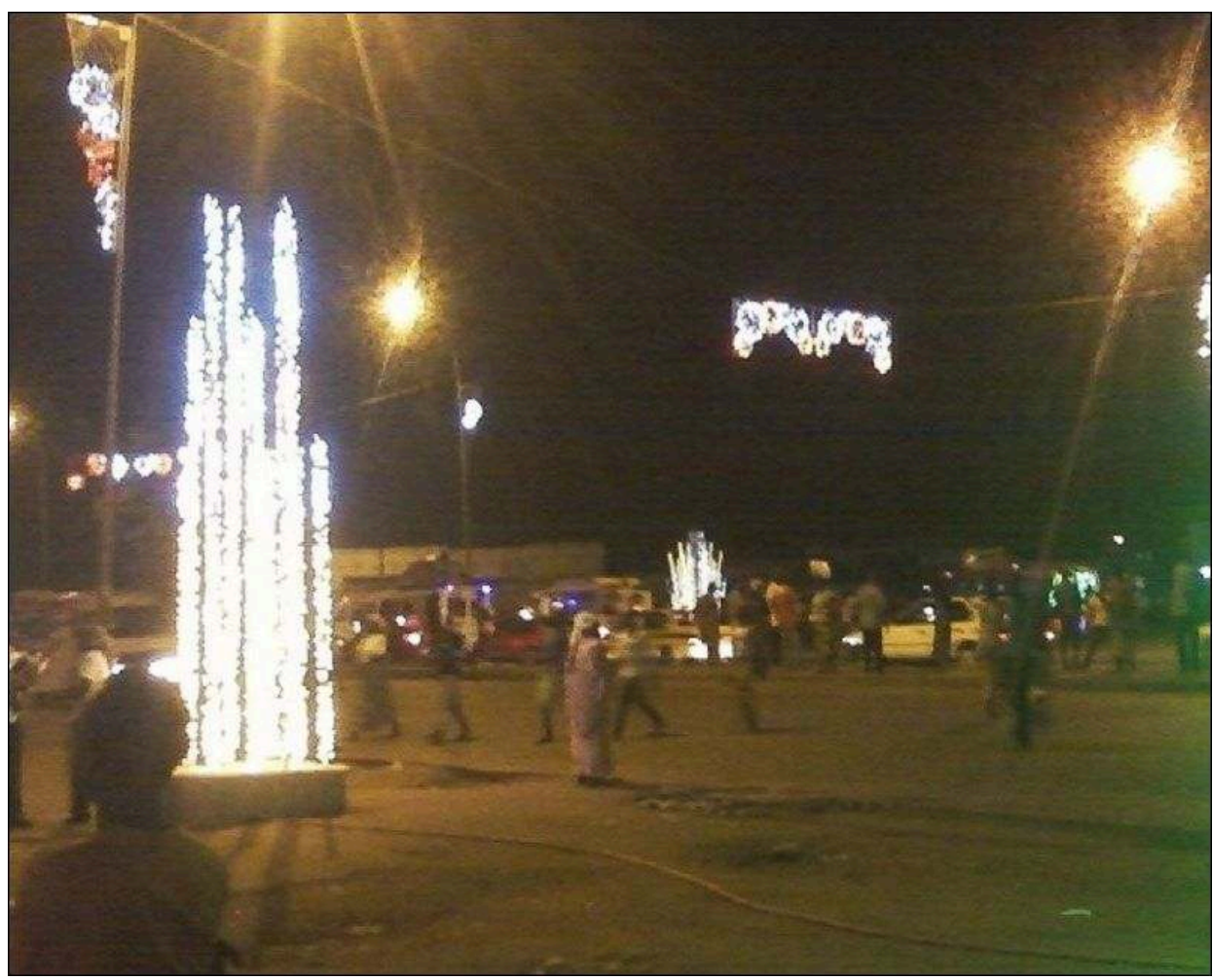

Au-delà de l'expression politique, la municipalité d'Abobo a initié cette forme d'art urbain dans l'optique de valoriser son territoire communal.

Auteur : I. Sagnon, 2012.

Les installations lumineuses initiées par le maire d'Abobo se caractérisent par l'utilisation de vis, de barres de fer, de petites ampoules, de fils électriques couverts par des tubes en plastique d'environ dix centimètres de diamètre et de régulateurs d'intensité électrique. En effet, les barres de fer sont fixées au sol par des vis. Sur ces barres de fer, sont disposées les ampoules donnant plusieurs formes et reliées entre elles par des fils électriques connectés au branchement des lampadaires situés en bordure de route. Une fois alimentées en électricité, les ampoules de couleur unique ou multicolores s'illuminent et laissent paraître diverses formes agréables au regard. Même de courte durée, l'installation lumineuse engendre une attractivité territoriale et contribue à l'épanouissement de la population.

\section{Conclusion}

La commune d'Abobo a connu diverses formes d'art urbain au cours des vingt dernières années. Originellement fondé sur une revendication des élèves face à des troubles dans l'éducation après le putsch militaire en 1999, le street art connaît une évolution. Au gbôchôli de ces élèves sur des murs de bâtiments publics en 2000, succède le graffiti en scénettes des étudiants peintres de l'INSAAC sur demande de l'USAID en 2010, année d'élection présidentielle. Ces étudiants utilisent leur art pour sensibiliser les citoyens dans l'optique de retrouver la cohésion nationale. Ces logiques socio-artistiques nées dès les premiers instants des troubles et de la campagne électorale confirment la place de premier rang de la jeunesse dans la construction de la citoyenneté via le street art. 
Toutefois, survint la crise post-électorale qui a enregistré des pertes en vie humaine notamment parmi les partisans du RDR à Abobo. Appartenant au même parti politique, le premier magistrat de la commune initie des installations lumineuses pour véhiculer des messages. En 2012, 2013 et 2014, il s'engage non seulement à rendre hommage aux martyres de la crise post-électorale, mais aussi à valoriser le territoire communal. Ainsi, force est de conclure que ces différentes logiques qui sous-tendent l'institutionnalisation du street art à Abobo sont intimement liées à la chronologie du contexte politique ivoirien.

\section{BIBLIOGRAPHY}

Arts Visuels, 2015. Le street art ou art urbain. [En ligne], 21 p. https://wheb.ac-reims.fr/dsden10/ exper/IMG/pdf/le_street_art.pdf

Babo, A., 2006. L'immigration et la citoyenneté au cœur du jeu ivoirien. Géopolitique africaine, $\mathrm{n}^{\circ}$ 22 , p. $165-173$.

Babo A., 2009. Faillite de l'État et administration de l'espace public politique par les jeunes en Côte d'Ivoire. Afrique et développement, vol. XXXIV, $\mathrm{n}^{\circ} 3$ \& 4, p. 27-45.

Bahi A., 2003. La «Sorbonne » d'Abidjan : rêve de démocratie ou naissance d'un espace public? Revue africaine de sociologie, vol. 7, $\mathrm{n}^{\circ}$ 1, p. 47-63.

Banégas R. et al. (dir.), 2012. Parlements de la rue : espaces publics de la parole et citoyenneté en Afrique. Politique africaine, $\mathrm{n}^{\circ} 127$.

Banégas R. (dir.), Marshall-Fratani R. (dir.), 2003. La Côte d'Ivoire en guerre : dynamiques du dedans et du dehors. Politique africaine, $\mathrm{n}^{\circ} 89$.

Bengtsen P., 2014. The Street Art World. Thèse de doctorat, Lund University, Division of Art History and Visual Studies, Department of Arts and Cultural Sciences, 229 p.

Blanché U., 2015. Qu'est-ce que le street art ? Essai et discussion des définitions. Cahiers de Narratologie [En ligne], n² 29. http://journals.openedition.org/narratologie/7397

BNF (Bibliothèque Nationale de France), 2016. Droit(s) et street art. Bibliographie sélective. 8 p. http://www.bnf.fr/documents/biblio_droit_street_art.pdf

Bottani E., 2016. Du tag au street art, quand l'art urbain devient un outil de requalification urbaine. Une approche comparée des villes de Marseille et Montréal. Mémoire de master en Urbanisme, AixMarseille Université, 168 p.

Catz J., 2013. Street art. Paris, Flammarion, 255 p.

Colas C., 2010. Paris Graffiti : Les marques secrètes de l'histoire. Paris, Parigramme, 144 p.

Couret D., 1996. Entre environnement urbain et développement local à Abidjan, pour une nouvelle mise en perspective de la ville du Sud. In : Villes du Sud. Sur la route d'Istanbul. Paris, Orstom Éditions, p. 429-434. 
Cutolo A., 2014. Le retour de Gbagbo. Jeunes Patriotes, herméneutique de l'histoire et subjectivation religieuse. In F. Vitti (dir.), La Côte d'Ivoire, d'une crise à l'autre. Paris, L'Harmattan.

Denys R., 2000. Qu'est-ce que l'art moderne ? Paris, Gallimard, p. 324-325.

Fedel A., 2013. Le street art à l'heure de son institutionnalisation [En ligne]. 14 p. http://docplayer.fr/ 12728029-Le-street-art-a-l-heure-de-son-institutionalisation.html

Fontaine B., 2014. Découvrir et Comprendre le graffiti : des origines à nos jours. Paris, Eyrolles, $160 \mathrm{p}$.

Gorri E., 2011. Le street art : une appropriation de l'espace public par l'imagerie contestataire. M2 CPP ISIC/Science Po Bordeaux, Bordeaux, p. 1-12.

Heritier A., 2014. Le street art, bien commun artistique ? Juris art, $\mathrm{n}^{\circ} 12$, p. 39-41.

La valette D., 2005. Keith Haring : quand l'art descend dans la rue. Paris, Palette, p. 20-24.

Lemoine S., 2012. L'art Urbain, du graffiti au street-art. Paris, Gallimard, 128 p.

Lionel R., 2002. L'aventure de l'art contemporain de 1945 à nos jours. Paris, Chêne, 256 p.

Mayer M., (dir), 2010. Basquiat. Paris, Flammarion, 224 p.

Mazenc L., 2015. Le street-art, ou l'institutionnalisation problématique d'une pratique contre-culturelle. Mémoire de master 1 RES, Département de Sociologie, Université Toulouse Jean Jaurès, 101 p.

Réseau des Médiathèques du Val d'Europe, 2012. Dossier pédagogique sur le thème de "l'art dans la rue ». Val d'Europe, médiathèque de Serris, $33 \mathrm{p}$.

Yang C., 2014. Graffiti et street art : étude des discours historiographiques et de la critique esthétique d'une forme sociale de modernité visuelle. Thèse de doctorat, Université de Grenoble, 540 p.

\section{NOTES}

1. La Sorbonne : «Convivial et divertissant, un espace public où l'on supporte mieux le poids de la mi-journée et l'on oublie surtout le chômage, la galère et toutes sortes de difficultés. Située en plein cœur du quartier des affaires du Plateau, la Sorbonne est partie d'un espace de causerie entre midi et deux pour devenir une sorte d'agora où les problèmes sociaux qui gangrènent la Côte d'Ivoire avaient pignons sur rue. [...] Au fur et à mesure que la Sorbonne gagnait en notoriété, les politiciens s'y intéressaient » (Banégas R., 2012, p. 22).

2. La FESCI : Fédération Estudiantine et Scolaire de Côte d'Ivoire fut, avec le FPI, l'un des acteurs majeurs de la revendication démocratique dans les années 1990, avant de se muer en véritable appareil de terreur sur les campus et de devenir l'une des principales milices du régime de Gbagbo (Banégas R., 2012).

3. Dans le souci de ne pas être perturbé dans nos prises de vue, les photos ont donc été prises, juste avant l'arrivée massive de la population, aux environs de 19 heures. 


\section{ABSTRACTS}

Street art or urban art is a contemporary artistic movement which brings together all forms of art achieved in streets and public spaces. Since 2000, street art has become young people's customs in Côte d'Ivoire. Over years, urban art is spreading to reach governments and becomes institutionalized with the implication of the commune of Abobo. Originally, street art gave public spaces a precarious image. It periodically contributes to the valuation of communal territory in its institutionalization phase. From these remarks, it results the problem of illegal appropriation of public spaces through the forms of art. The study aims at revealing the logics which underlie street art institutionalization in the commune of Abobo. This work results in the construction of a citizenship and politicization trajectories through the channel of urban art.

Le street art ou l'art urbain est un mouvement artistique contemporain qui regroupe toutes les formes d'art réalisées dans les rues et espaces publics. En Côte d'Ivoire, depuis l'année 2000, le street art est entré dans les mœurs des jeunes. Au fil des années, l'art urbain se repend jusqu'atteindre des gouvernants et s'institutionnalise avec l'implication de la commune d'Abobo. À l'origine, le street art donnait une image précaire aux espaces publics. Dans sa phase d'institutionnalisation, il contribue de façon périodique à la valorisation du territoire communal. De ces constats, se dégage le problème d'appropriation illégale d'espaces publics à travers des formes d'art. L'étude vise à révéler les logiques qui sous-tendent l'institutionnalisation du street art dans la commune d'Abobo. Il résulte de cette étude, la construction d'une citoyenneté et des trajectoires de politisation par le canal de l'art urbain.

\section{INDEX}

Keywords: Abobo, valuation, public space, street art, institutionalization

Mots-clés: Abobo, valorisation, espace public, street art, institutionnalisation

\section{AUTHORS}

\section{IBRAHIMA SAGNON}

Ibrahima Sagnon, ibrahimasagnon3@gmail.com, est doctorant en Géographie, Université Alassane Ouattara (Côte d'Ivoire). Il a notamment co-écrit :

- Sagnon I., Ouattara Teninan H., Béchi Grah F., 2018. Analyse de l'assainissement dans l'espace urbain de Bouaké (Côte d'Ivoire). Espaces, sociétés et développement en Afrique subsaharienne, tome 2. Lomé, Presses Universitaires de Lomé, p. 503-521.

\section{HUGUES OUATTARA TENINAN}

Hugues Ouattara Teninan, ouattarateninan@gmail.com, est doctorant en Géographie, Université Alassane Ouattara (Côte d'Ivoire). Il a notamment co-écrit :

- Sagnon I., Ouattara Teninan H., Béchi Grah F., 2018. Analyse de l'assainissement dans l'espace urbain de Bouaké (Côte d'Ivoire). Espaces, sociétés et développement en Afrique subsaharienne, tome 2. Lomé, Presses Universitaires de Lomé, p. 503-521. 


\section{FÉLIX BÉCHI GRAH}

Félix Béchi Grah, felixbechi@yahoo.fr, est maître de Conférences en Géographie, Université Alassane Ouattara (Côte d'Ivoire). Il a notamment publié :

- Sagnon I., Ouattara Teninan H., Béchi Grah F., 2018. Analyse de l'assainissement dans l'espace urbain de Bouaké (Côte d'Ivoire). Espaces, sociétés et développement en Afrique subsaharienne, tome 2. Lomé, Presses Universitaires de Lomé, p. 503-521.

- Béchi Grah F, 2013. La gestion des eaux usées dans les villes forestières ivoirières : des risques de marginalité. Revue de Géographie du Laboratoire Leidi, ${ }^{\circ} 11$, p. 161-178.

- Béchi Grah F, 2009. Urbanisation et risques environnementaux à Abidjan. Le journal des sciences sociales, $\mathrm{n}^{\circ} 6$, p. 67-82. 